\title{
Transport of particles suspended within a temperature-dependent viscosity fluid using coupled LBM-DEM
}

\author{
J.W.S. McCullough ${ }^{\mathrm{a}, *}$, S.M. Aminossadati ${ }^{\mathrm{a}}$, C.R. Leonardi ${ }^{\mathrm{a}}$ \\ ${ }^{a}$ The University of Queensland, School of Mechanical and Mining Engineering, Cooper \\ Road, St Lucia QLD 4072, Australia
}

\begin{abstract}
Particle suspensions are present in a wide variety of practical settings. Modelling these numerically is a challenging task that often requires the combination of multiple methodologies. This paper examines particle transport within a temperature-dependent viscosity fluid utilising a coupled approach of the lattice Boltzmann method and the discrete element method. This technique takes advantage of the locality of the lattice Boltzmann method to allow both the individual particle behaviour to be fully resolved and to permit fine-scale variation of fluid viscosity throughout the tested domains. It is firstly shown that a total energy conserving form of the lattice Boltzmann method is needed to accurately reconstruct the non-linear temperature profiles observed on Couette flows of fluids with changing viscosity. This model is then coupled to the discrete element method to demonstrate the quantitative and qualitative changes to particle motion that arise in channel-based geometries in the presence of a temperature-dependent viscosity fluid exposed to a constant temperature gradient. In particular, it is demonstrated that the particles settled faster in such and appear less likely to deviate into side channels in the presence of such fluids. These results demonstrate that temperature-dependent viscosity requires special consideration to be simulated correctly and does have quantitative impact
\end{abstract}

\footnotetext{
* Corresponding author

Email addresses: jon.mccullough@uqconnect.edu.au (J.W.S. McCullough), s.aminossadati@uq.edu.au (S.M. Aminossadati), c.leonardi@uq.edu.au (C.R. Leonardi)
}

Preprint submitted to International Journal of Heat and Mass Transfer February 4, 2020 
on particle transport. This impact should be considered in models of fluids of changing temperature.

Keywords: Particle suspensions, Thermal lattice Boltzmann method, Discrete element method, Temperature-dependent viscosity

\section{Introduction}

In many scientific, engineering and industrial settings a working fluid is subjected to a thermal gradient. Such a gradient can be localised or distributed over a significant distance and may be the result of intentional heat transfer to 5 the fluid or a consequence of the operation of other components in the system. While all material properties of a fluid exhibit some degree of variation due to changing temperature, changing viscosity often presents the most obvious macroscopic examples of this. It is of interest then that in many studies this temperature-dependent variation is neglected in favour of a constant viscosity (e.g. [1 3]). While there may be some situations where this is an appropriate assumption to make, this is often not the case.

One situation where the influence of fluid viscosity is significant is in particle suspensions. When used in a practical setting, the motion and transport of the particles through the system is often the major focus of the application. Changing viscosity of the fluid phase in these situations consequently alters the hydrodynamic forces acting on the particles and, as such, the rate at which particles settle under gravity or are carried by the flow.

Given the change in material properties can be highly localised, the modelling technique used to capture such behaviour needs to be formulated in a manner that can accommodate this. The implementation approach of the lattice Boltzmann method (LBM) provides a local framework for performing such calculations in an efficient and robust manner. A number of existing studies use LBM to capture local changes in viscosity associated with non-Newtonian fluids 4 6] This indicates that similar strategies could be implemented in situations where viscosity changes are thermal in origin. The study of temperature- 
dependent viscosity within an LBM framework has previously been conducted or discussed in a relatively small number of prior works [7-10]. The current work seeks to firstly provide a more robust analysis of the performance of LBM models for calculating problems with temperature-dependent viscosity. Secondly, this will be extended to the modelling of particle suspensions to further examine how such changes impact on the individual and collective motion of particles within a fluid.

The remainder of this paper is structured as follows. Section 2 describes the numerical methods used for modelling in this research whilst Section 3 presents the verification of the model and the investigations conducted with it. The work is summarised in Section 4 .

\section{Details of numerical modelling}

To capture the behaviour of particle suspensions with thermal interactions, this study has developed a coupled approach between the LBM and the dis40 crete element method (DEM). In this approach, a two-population LBM model captures the fluid behaviour and the thermal interactions of the entire coupled system. The DEM is used to compute the physical motion of the solids and their interactions with the surrounds. When the temperature of a particle is required, the average temperature of the LBM nodes covered by the particle can be used to compute this quantity.

\subsection{Fluid model - lattice Boltzmann method}

Within the LBM, a discretised distribution function, $f_{i}(\mathbf{x}, t)$, is used to represent the probability of an amount of the considered quantity at a particular location, $\mathbf{x}$, moving in direction $i$ at a given point in time, $t$. Evolution of this population through time occurs through the discrete version of the lattice Boltzmann equation,

$$
f_{i}\left(\mathbf{x}+\mathbf{c}_{i} \Delta t, t+\Delta t\right)-f_{i}(\mathbf{x}, t)=\Omega_{i}\left(f_{i}(\mathbf{x}, t)\right),
$$


where $\mathbf{c}_{i}$ represents the velocity vector in direction $i$ and $\Omega_{i}\left(f_{i}(\mathbf{x}, t)\right)$ is the function representing collision and redistribution of the quantity. The most common choices of $\Omega_{i}$ in the lattice Boltzmann literature are based on the relaxation of the $f_{i}(\mathbf{x}, t)$ populations towards an equilibrium state, $f_{i}^{e q}(\mathbf{x}, t)$, using single, two or multiple relaxation times [11 15]. The single relaxation time version (SRT) is used in this paper due to its straightforward implementation and minimised number of tunable parameters. In this setting the collision function,

$$
\Omega_{i}\left(f_{i}(\mathbf{x}, t)\right)=-\frac{\Delta t}{\tau_{f}}\left(f_{i}(\mathbf{x}, t)-f_{i}^{e q}(\mathbf{x}, t)\right),
$$

is controlled by the relaxation parameter, $\tau_{f}$. Under the Chapmann-Enskog expansion process, $\tau_{f}$ can be related to the transport coefficient of the quantity being studied. For hydrodynamics this enables the definition of the fluid kinematic viscosity, $\nu$, to be related to the lattice spacing, $\Delta x$, and time step, $\Delta t$, through,

$$
\nu=\frac{1}{3}\left(\tau_{f}-\frac{1}{2}\right) \frac{(\Delta x)^{2}}{\Delta t}
$$

When solving in lattice units, $\Delta x$ and $\Delta t$ are respectively set to unity. When representing a physical quantity, the equilibrium function is often built upon a velocity-based Taylor expansion of the well known Maxwell distribution. This form,

$$
f_{i}^{e q}(\mathbf{x}, t)=w_{i} \rho(\mathbf{x}, t)\left(1+\frac{\mathbf{c}_{i} \cdot \mathbf{u}}{C_{s}^{2}}+\frac{\left(\mathbf{c}_{i} \cdot \mathbf{u}\right)^{2}}{C_{s}^{4}}-\frac{|\mathbf{u}|^{2}}{C_{s}^{2}}\right),
$$

contains a weighting constant, $w_{i}$, that is dependent on the velocity set chosen.

${ }_{60}$ In this work, the conventional D2Q9 and D3Q27 lattices have been used for all populations in $2 \mathrm{D}$ and $3 \mathrm{D}$ studies, respectively. Both of theses cases use $i=0$ to represent the location of interest. The remaining velocity directions are numbered according to the increasing distance to neighbour nodes on the conventional, evenly spaced, Cartesian grid of nodes used to represent the simu65 lation domain. Values for $w_{i}$ are also found as a result of the Chapmann-Enskog expansion. Using the common D2Q9 LBM lattice, these are $4 / 9$ for $i=0,1 / 9$ for $i=1,2,3,4$ and $1 / 36$ for $i=5,6,7,8[16$. In a D3Q27 setting, the groups of lattice directions from the current node use weights of $8 / 27,2 / 27,1 / 54$ and 
$1 / 216$ for each increasingly distant neighbour direction [17. The lattice speed of sound, $C_{s}^{2}$, is also found to be a value of $1 / 3$ from this process. The $\mathbf{u}$ term represents the velocity vector at the nodal location of interest. The zeroth moment of the population, $\rho(\mathbf{x}, t)=\sum_{i} f_{i}(\mathbf{x}, t)$, is taken to represent the density of the distribution functions at $\mathbf{x}$. Higher moments of a population can also represent physically meaningful macroscopic properties. A typical example of this is when flow. Here $f_{i}(\mathbf{x}, t)$ is related to mass, the first moment, $\rho(\mathbf{x}, t) \mathbf{u}=\sum_{i} f_{i}(\mathbf{x}, t) \mathbf{c}_{i}$, corresponds to momentum and the second moment is related to the viscous stress tensor $S_{\gamma \delta}(\mathbf{x}, t)=-\left(1-1 /\left(2 \tau_{f}\right)\right) \sum_{i} \mathbf{c}_{i \gamma} \mathbf{c}_{i \delta}\left(f_{i}(\mathbf{x}, t)-f_{i}^{e q}(\mathbf{x}, t)\right)$.

\subsubsection{Thermal lattice Boltzmann methods}

As the energy of a fluid system can be described with a convection-diffusion equation, the LBM can again be used to solve this behaviour within a suspension. The most common approach for doing this is for temperature, $T(\mathbf{x}, t)$, to be the subject of a convection-diffusion equation and the quantity conserved by the zeroth moment of the LBM population. This is referred to as the passive scalar approach. The formulation of this model in LBM is very similar to that presented for the fluid case. For distinction, the second population will be referred to as $g_{i}(\mathbf{x}, t)$. An SRT scheme is again used for the evolution of the population,

$$
g_{i}\left(\mathbf{x}+\mathbf{c}_{i} \Delta t, t+\Delta t\right)-g_{i}(\mathbf{x}, t)=-\frac{\Delta t}{\tau_{g}}\left(g_{i}(\mathbf{x}, t)-g_{i}^{e q}(\mathbf{x}, t)\right),
$$

with $\tau_{g}$ being the associated relaxation parameter and $\sum_{i} g_{i}(\mathbf{x}, t)=T(\mathbf{x}, t)$. The equilibrium function is calculated by,

$$
g_{i}^{e q}(\mathbf{x}, t)=w_{i} T(\mathbf{x}, t)\left(1+\frac{\mathbf{c}_{i} \cdot \mathbf{u}}{C_{s}^{2}}+\frac{\left(\mathbf{c}_{i} \cdot \mathbf{u}\right)^{2}}{C_{s}^{4}}-\frac{|\mathbf{u}|^{2}}{C_{s}^{2}}\right),
$$

using the velocity calculated from the fluid population. The thermal equilibrium function uses the same weights as $f_{i}^{e q}(\mathbf{x}, t)$. The thermal diffusivity of the fluid, 
$\alpha$, is again related to the lattice spacing and time step through,

$$
\alpha=\frac{1}{3}\left(\tau_{g}-\frac{1}{2}\right) \frac{(\Delta x)^{2}}{\Delta t} .
$$

As acknowledged by $\mathrm{He}$ et al. 2, this passive scalar approach is unable to capture thermally related processes of viscous heating and compression work. To overcome this, they proposed a model conserving internal energy instead of temperature. Non-local data requirements in the calculation procedure meant that this model was not suited to modelling more general flow problems [1]. A total energy conserving model was proposed by Guo et al. 8] that was able to overcome this obstacle.

In this model the quantity conserved by the $g_{i}$ population is total energy, $E(\mathbf{x}, t)=c T(\mathbf{x}, t)+0.5|\mathbf{u}|^{2}$, such that

$$
\sum_{i} g_{i}(\mathbf{x}, t)=\rho(\mathbf{x}, t) E(\mathbf{x}, t)-\frac{\Delta t}{2} \mathbf{u} \cdot \mathbf{a} .
$$

where $c$ is the heat capacity of the fluid and $\mathbf{a}$ is the acceleration vector for any external forces acting on the system. The only variation to the $f_{i}$ population is for it to be relaxed by,

$$
f_{i}\left(\mathbf{x}+\mathbf{c}_{i} \Delta t, t+\Delta t\right)-f_{i}(\mathbf{x}, t)=-\omega_{f}\left(f_{i}(\mathbf{x}, t)-f_{i}^{e q}(\mathbf{x}, t)\right)+\frac{2-\omega_{f}}{2} \Delta t F_{i},
$$

where $\omega_{f}=\frac{2 \Delta t}{2 \tau_{f}+\Delta t}$ and $F_{i}$ is an external mechanical forcing term computed by,

$$
F_{i}(\mathbf{x}, t)=w_{i} \rho(\mathbf{x}, t)\left(\frac{\mathbf{c}_{i} \cdot \mathbf{a}}{C_{s}^{2}}+\frac{\left(\mathbf{c}_{i} \cdot \mathbf{a}\right)\left(\mathbf{c}_{i} \cdot \mathbf{u}\right)}{C_{s}^{4}}-\frac{\mathbf{a} \cdot \mathbf{u}}{C_{s}^{2}}\right) .
$$

For the $g_{i}(\mathbf{x}, t)$ population, $\omega_{g}=\frac{2 \Delta t}{2 \tau_{g}+\Delta t}$ and the SRT relaxation function becomes,

$g_{i}\left(\mathbf{x}+\mathbf{c}_{i} \Delta t, t+\Delta t\right)-g_{i}(\mathbf{x}, t)=-\omega_{g}\left(g_{i}(\mathbf{x}, t)-g_{i}^{e q}(\mathbf{x}, t)\right)+\frac{2-\omega_{g}}{2} \Delta t q_{i}(\mathbf{x}, t)+\left(\omega_{g}-\omega_{f}\right) Z_{i}(\mathbf{x}, t) \Phi_{i}(\mathbf{x}, t)$.

in which:

$$
Z_{i}(\mathbf{x}, t)=\mathbf{c}_{i} \cdot \mathbf{u}-\frac{|\mathbf{u}|^{2}}{2}
$$




$$
\Phi_{i}(\mathbf{x}, t)=f_{i}(\mathbf{x}, t)-f_{i}^{e q}(\mathbf{x}, t)+\frac{\Delta t F_{i}(\mathbf{x}, t)}{2}
$$

and

$$
q_{i}(\mathbf{x}, t)=w_{i} \frac{\rho(\mathbf{x}, t) E(\mathbf{x}, t)}{C_{s}^{2}}+f_{i}(\mathbf{x}, t) \mathbf{c}_{i} \cdot \mathbf{a} .
$$

$g_{i}^{e q}(\mathbf{x}, t)=w_{i} \rho(\mathbf{x}, t) C_{s}^{2}\left[\frac{\mathbf{c}_{i} \cdot \mathbf{u}}{C_{s}^{2}}+\frac{\left(\mathbf{c}_{i} \cdot \mathbf{u}\right)^{2}}{C_{s}^{4}}-\frac{u^{2}}{2 C_{s}^{2}}+\frac{1}{2}\left(\frac{\mathbf{c}_{i}^{2}}{C_{s}^{2}}-N\right)\right]+E(\mathbf{x}, t) f_{i}^{e q}(\mathbf{x}, t)$,

where $N$ is the dimensionality of the lattice being studied.

\subsection{Solid model - discrete element method}

The discrete element method is an approach for computing the motion of solids that treats individual components of material as point particles. By com-

105

bining knowledge of the physical properties of a particle and the forces acting upon it, numerical integration of Newton's second law allows the kinematic state of the particle to be explicitly updated through time. Due to their geometric simplicity in 3D, spheres (circles in 2D) are the most widely-used particle representation for individual elements [18. More complicated bodies can be described by bonding a collection of individual particles together. The nature of this bond can allow the DEM to model both rigid and deformable bodies [19. If a more rigorous implementation of non-spherical particles is desired then similar principles can be employed with an appropriate contact model [20].

In terms of algorithmic implementation, the DEM can be broken into four basic steps [18, 21]:

1. Contact search - Identification of particle pairs that may be in contact.

2. Contact resolution - Computation of the small overlap between particle pairs identified in the search step.

3. Force resolution - Computation of the forces acting on individual particles. These may arise from sources including physical contact, electrostatic/magnetic effects, lubrication and gravitational effects. 
4. Kinematic integration - Numerical integration of Newton's second law using the calculated forces and associated torques (defined by particle geometry) to update the kinematic state of the particle.

$$
f_{i}\left(\mathbf{x}+\mathbf{c}_{i} \Delta t, t+\Delta t\right)-f_{i}(\mathbf{x}, t)=-\frac{\Delta t}{\tau_{f}}[1-B]\left(f_{i}(\mathbf{x}, t)-f_{i}^{e q}(\mathbf{x}, t)\right)+B \Omega_{i}^{s} .
$$

Here $B$ represents the fraction of the unit cell surrounding an LBM node that is covered by a solid particle, as highlighted in Figure 1, and $\Omega_{i}^{s}$ represents a collision function for interactions between the LBM population and the solid particles. This work uses the non-equilibrium bounceback approach proposed

In this work, each suspended particle is represented by a single circular or spherical DEM particle. The forces acting on the particles arise from physical contact, gravity and hydrodynamics only.

\subsubsection{Fluid-Particle Coupling}

Coupling the behaviours of the fluid and solid models require two actions fluid model such that the relaxation can be appropriately modified as needed. Additionally, the hydrodynamic forces acting on the particles, as calculated from the fluid model, needs to be communicated to the solids. Within an LBM-DEM framework, a number of approaches have been proposed, including models by Ladd [22, 23, Suzuki and Inamuro 24], and Noble and Torczynksi 25]. Similar work has been applied to elliptical particles by Xu et al. [26] and Walayat et al. [20].

In this work, the partially saturated method (PSM) of Noble and Torczynski has been used to couple the LBM and DEM components. In this approach, the relaxation of the fluid LBM population is modified according to, by Noble and Torczynksi 25. that utilises the particle velocity, $\mathbf{u}_{p}$,

$$
\Omega_{i}^{s}=f_{-i}(\mathbf{x}, t)-f_{i}(\mathbf{x}, t)+f_{i}^{e q}\left(\rho, \mathbf{u}_{p}\right)-f_{-i}^{e q}(\rho, \mathbf{u}) .
$$




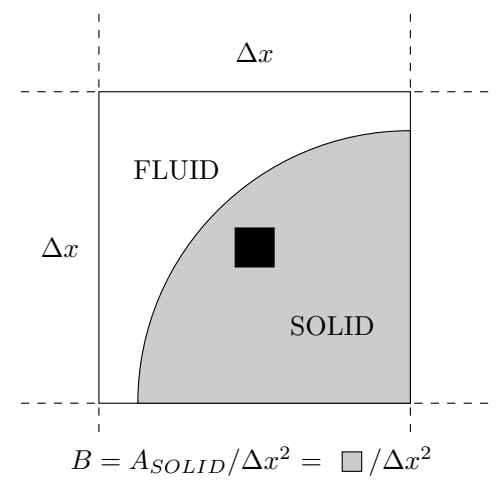

Figure 1: Strategy for calculating the nodal coverage fraction $B$ for use in the PSM method. Interface nodes for particle boundaries are identified as $B \in[0,1]$. Note that the solid and dotted lines in this figure are offset from the lattice grid linking nodes by $\Delta x / 2$.

The hydrodynamic force and torque acting on a particle centred at $\mathbf{x}_{p}$ can be, respectively, found by [21],

$$
\mathbf{F}_{f}=\frac{\Delta x^{2}}{\Delta t} \sum_{r} B_{r}\left(\sum_{i} \Omega_{i}^{s} \mathbf{c}_{i}\right),
$$

and,

$$
\mathbf{T}_{f}=\frac{\Delta x^{2}}{\Delta t} \sum_{r}\left(\mathbf{x}-\mathbf{x}_{p}\right) \times B_{r}\left(\sum_{i} \Omega_{i}^{s} \mathbf{c}_{i}\right) .
$$

In these expressions, $r$ counts the nodes that map an obstacle to the lattice. This explicit resolution of forces acting on a particle classifies the current model as a particle-resolved method under the classification scheme outlined by $\mathrm{Xu}$ et al. 27.

\subsubsection{Calculation of particle coverage}

Central to the PSM is computing the quantity, $B$ (see Figure 1). In stationary geometries, this is often straightforward and only needs to be performed during the initialisation of a simulation. For example, it can be achieved through application of an analytic description of a domain. Transient problems such as particle suspensions require this term to be recomputed regularly throughout a simulation. In $3 \mathrm{D}$, this is most tractable for rigid spheres but can still be a 
time-consuming task. Such approaches for computing this factor include Monte Carlo approximations, sub-division of lattice cells, edge intersection averaging or calculation of convex hulls 28. Recently, Jones and Williams 28 proposed a linear approximation technique that significantly outperforms these methods both in terms of computation time for equivalent accuracy and accuracy for given computation time. They recognise that when mapping a sphere that is larger than an LBM cell, the coverage of a majority of cells varies linearly with the distance to the centre of the sphere. In their method, the coverage of a node-centred cell by a sphere, with radius $r$, is found through,

$$
B=-\hat{D}+V_{a}-R+0.5
$$

where $\hat{D}$ is the distance between the node and the surface of the sphere and $R=r / \Delta x$ is the sphere radius normalised by the grid spacing. The $V_{a}$ term is the volume of the sphere contained within the cell. The authors present the solution to the associated integral as,

$V_{a}=\left(\frac{1}{12}-R^{2}\right) \arctan \left(\frac{A}{1-2 R^{2}}\right)+\frac{A}{3}+\left(R^{2}-\frac{1}{12}\right) \arctan \left(\frac{1}{2 A}\right)-\frac{4 R^{3}}{3} \arctan \left(\frac{1}{4 R A}\right)$,

where $A=\sqrt{R^{2}-0.5}$. The value of $A$ is constant for a given particle size and may be pre-computed prior to a simulation. The authors also give an equivalent expression for calculating the coverage of circles in 2D simulations. In practice, suspensions may be studied where multiple particle sizes are present. As such, when performing a calculation on a particle, $V_{a}$ is not known a priori. From analysis of the function for $V_{a}$ over a wide range of $R$, it was recognised that Equation 21 could be sufficiently approximated as,

$$
V_{a} \approx R-K / R
$$

Here, $K$ is a constant that was taken as 0.084 for $3 \mathrm{D}$ and noted to be 0.042 for the $2 \mathrm{D}$ case. In particular, this result is valid for $R>2$. This is an acceptable outcome as larger values for $R$ are needed for correct hydrodynamic behaviour 
to be computed. This means that the coverage of a cell by a sphere can be computed solely based on its distance from the centre of any given particle in the simulation as,

$$
B=-\hat{D}-K / r+0.5
$$

As in the work by Jones and Williams, this value is capped such that $B \in[0,1]$ is

maintained. For the results in the following section, only LBM nodes that were centred within a particle had their coverage mapped. This was found to yield superior results in the tests conducted for the particle resolution of interest.

\subsection{Terminal velocity of a single particle between parallel plates}

To demonstrate that the hydrodynamics of a particle within a fluid is correctly captured by a 3D model, the test case of a particle settling between two parallel plates was used. In this scenario, a particle was placed in a stationary fluid between two plates and accelerated from rest by a body force with the terminal velocity of the particle used as an evaluative measure. In the low Reynolds number regime of Stokes flow (i.e. $R e \ll 1$ ), the velocity of the particle is given by Wang et al. 29] as,

$$
U_{\text {sphere }}=\frac{F\left(1-0.625(r / l)+0.1475(r / l)^{3}-0.131(r / l)^{4}-0.0644(r / l)^{5}\right)}{6 \pi \rho \nu r} .
$$

Here, $r$ is the radius of the sphere and $l$ its distance from the nearest wall. $F$ is the magnitude of the force applied to the particle.

For the test case presented here (see Figure 2), the density of the particle was taken as $\rho=2,000 \mathrm{~kg} / \mathrm{m}^{3}$, and the particle was sized and placed such that $r / l=0.5$. The fluid possessed a kinematic viscosity of $\nu=10^{-6} \mathrm{~m}^{2} / \mathrm{s}$. A constant acceleration of $\mathbf{a}=0.01 \mathrm{~m} / \mathrm{s}^{2}$ was applied to the particle as the driving force parallel to the plates. This leads to a predicted terminal particle velocity of approximately $U_{\text {sphere }}=3.1 \times 10^{-5} \mathrm{~m} / \mathrm{s}$ and a particle Reynolds number of about 0.01 . Figure 3 shows the absolute relative error of the measured terminal 


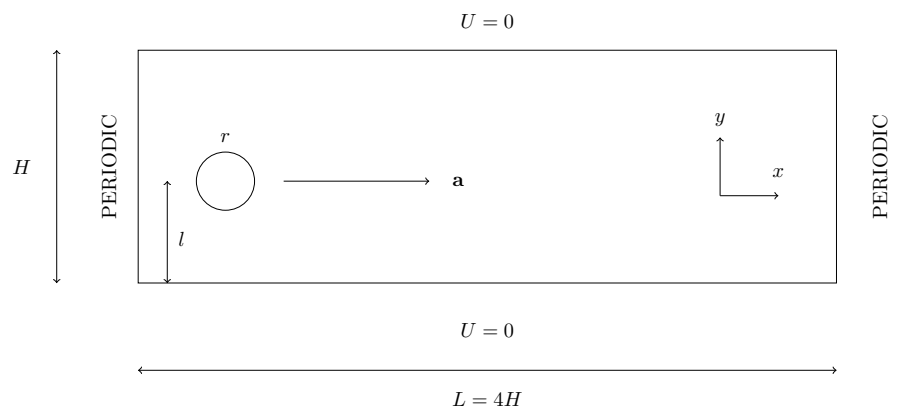

Figure 2: Mid-plane view of terminal velocity test used to verify the model's hydrodynamics. The model is periodic in the out-of-page direction which also had a total depth of $4 H$. The particle of radius $r=0.0001 \mathrm{~m}$ was placed such that $r / l=0.5$, while $l=H / 4$ was used in testing.

particle velocity. The results converge to this value at approximately order 2.8 with increasing radial resolution. The convergence of the model being greater than second order is consistent with previous data presented for this test case [29, 30]. These authors suggest the approximations inherent in the analytical solution as a possible cause for this. In the remainder of this work, a resolution of $r / \Delta x=6.4$ for free moving particles has been chosen as a compromise between resolving the hydrodynamics and computational speed. From Figure 3. the absolute relative error of the particle velocity of this case is approximately $1 \%$. In particular, this highlights that the PSM requires a minimum resolution of particle radius compared to grid spacing to accurately capture the hydrodynamics of a sphere moving through a viscous fluid.

\section{Numerical investigation of temperature-dependent flows}

The numerical investigations undertaken with this model have been broken into two parts. The first section verifies the performance of D2Q9 thermal LBM models against analytical results for temperature-dependent flows. The second section examines the transport of single and multiple particles through a fluid with varying viscosity. 


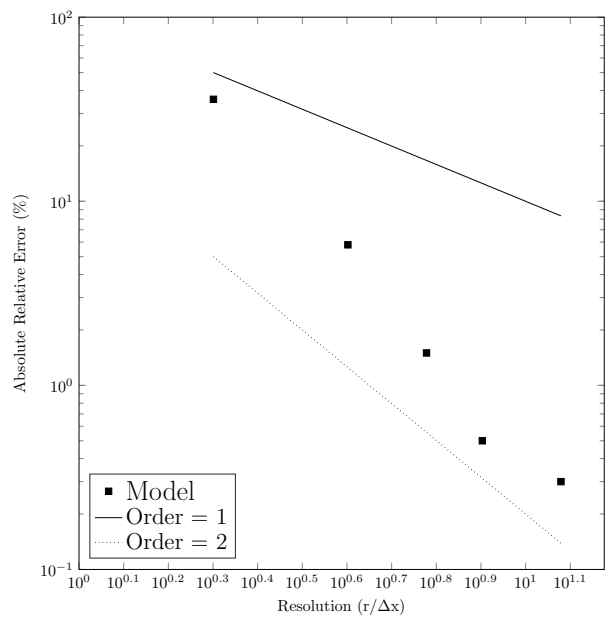

Figure 3: Convergence of the terminal velocity of a particle moving between parallel plates due to a constant acceleration. Slopes of first and second order convergence have been included for reference. The observed convergence order of approximately 2.8 is consistent with that observed in previous studies [29, 30].

\subsection{Model verification}

The work of Myers et al. 31] developed analytical expressions for the velocity and temperature profiles generated for a temperature-dependent viscosity fluid within a Couette flow experiencing an applied temperature gradient. The layout of the channel used to compare the model performance against these results is presented in Figure 4. In these tests, the dynamic viscosity of the fluid is assumed to vary in an exponential fashion with respect to temperature,

$$
\mu=\mu_{0} e^{-\beta T},
$$

where $\mu_{0}$ is a reference viscosity and $\beta$ is a coefficient indicating the degree of temperature-dependence. This form is chosen as it allows for an analytic solution. In practice though, this dependence could be implemented with any suitable function of temperature. The velocity profile is given in a normalised 
form by,

$U(y)=\sqrt{\frac{2 e^{\beta T_{m}}}{\beta B r}}\left\{\tanh \left[y \sqrt{\frac{A^{2} \beta B r}{2 e^{-\beta T_{m}}}}-\tanh ^{-1} \sqrt{1-e^{-\beta T_{m}}}\right]+\sqrt{1-e^{-\beta T_{m}}}\right\}$,

and the normalised (or dimensionless) temperature profile by,

$$
T(y)=T_{m}+\frac{1}{\beta} \ln \left\{1-\tanh ^{2}\left[\tanh ^{-1}\left(\sqrt{1-e^{-\beta T_{m}}}\right)-y \sqrt{\frac{A^{2} \beta B r}{2 e^{-\beta T_{m}}}}\right]\right\}
$$

The terms $T_{m}$ and $A$ are found through application of the boundary conditions

at the upper wall (normalised value of $y=1$ ). Typically this requires these equations to be solved numerically. Here, the normalised temperature can be calculated from a physical temperature through,

$$
T=\frac{T^{\text {physical }}-T_{C}^{\text {physical }}}{T_{H}^{\text {physical }}-T_{C}^{\text {physical }}},
$$

where $T_{H}^{\text {physical }}$ and $T_{C}^{\text {physical }}$ are, respectively, the reference hot and cold temperature of the system. For physically sensible results, these should be suitable to the system being considered (e.g. such that the Boussinesq approximation of small temperature differences still applies). In the following sections these may be considered to be $T_{H}^{\text {physical }}=350 \mathrm{~K}$ and $T_{C}^{\text {physical }}=300 \mathrm{~K}$. Additionally, the non-dimensional Brinkman number of the flow can be defined as,

$$
B r=\frac{\mu_{0} U_{\max }^{2}}{k\left(T_{H}-T_{C}\right)},
$$

using $k$ as the thermal conductivity of the fluid, $U_{\max }$ the velocity of the shearing wall and $T_{H}$ and $T_{C}$ the respective dimensionless temperatures of the moving (hot) and stationary (cold) walls. This term relates to the amount of viscous heat heating generated (e.g. by shearing flow) in the system to that transported by conduction.

To evaluate the performance of the LBM models a number of test cases with different values of $B r$ and $\beta$, as presented in Table 1, were conducted. The 


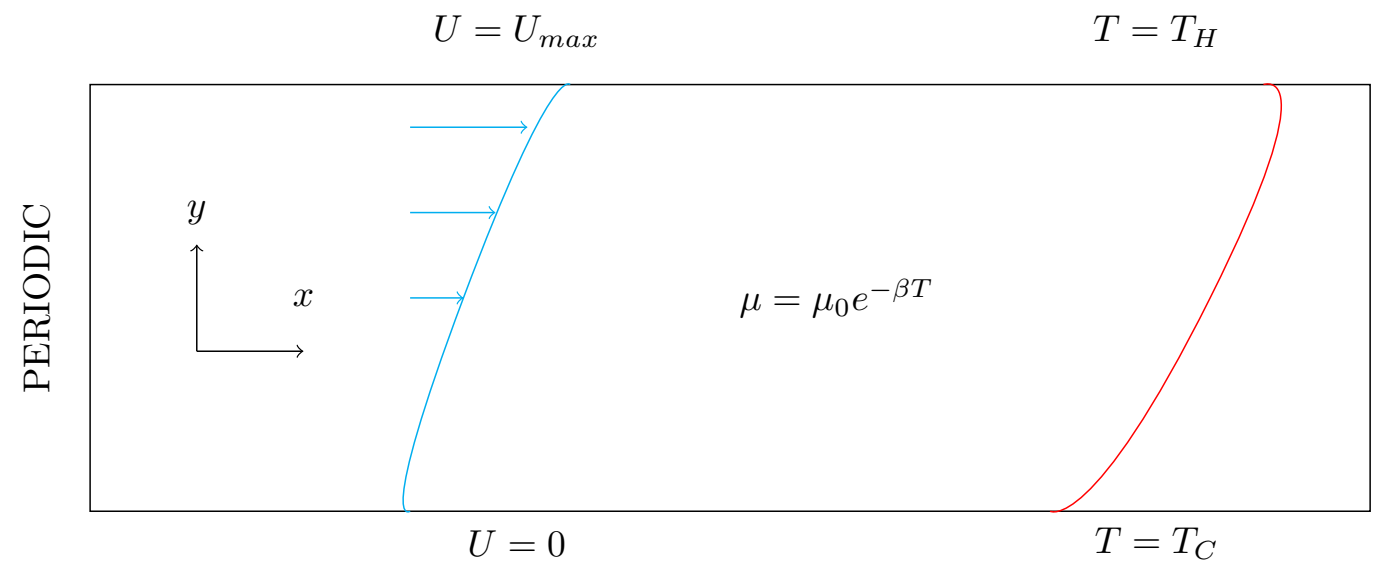

家

Figure 4: The Couette flow with temperature gradient used to test the numerical model of temperature-dependent viscosity. The flow is driven by an applied velocity of $U_{\max }$ at the upper wall whilst the lower wall is held stationary. The upper and lower wall temperatures are set to $T_{H}$ and $T_{C}$ respectively. The domain is periodic in the x-direction for both hydrodynamics and thermodynamics. Bulk fluid begins at rest at $T_{H}$. Analytical expressions for the velocity and temperature profiles across the height of the channel were developed by Myers et al. [31. 


\begin{tabular}{|c|c|c|}
\hline Parameter & $B r$ & $\beta$ \\
\hline Case 1 & 0.7 & 1 \\
Case 2 & 0.7 & 1.25 \\
Case 3 & 2 & 1 \\
Case 4 & 0.275 & 1 \\
Case 5 & 0.7 & 0.1 \\
\hline
\end{tabular}

Table 1: Parameter values used for the assessing the performance of the LBM models at capturing temperature-dependent viscosity flow behaviour by comparison to analytic results.

reference lattice viscosity was set at a value of $1 / 6$, with this being set at the cold (lower) wall. Note that lattice values are calculated by setting $\Delta x$ and $\Delta t=1$. From a numerical point of view, the local viscosity of the LBM population was varied using the exponential relationship based on the local LBM temperature as in Equation 25. This is represented by modifying the local value of $\tau_{f}$ while holding $\Delta t$ constant. As an incompressible fluid is being studied, the conversion between dynamic and kinematic viscosities is valid.

The height of the channels was set to $10 \Delta x$ for the coarsest grid spacing test. This was then increased to achieve a medium $(20 \Delta x)$ and fine $(40 \Delta x)$ grid spacing. The fixed temperature and velocity boundary conditions were implemented using the non-equilibrium extrapolation method similar to those described in Guo et al. [8] and Frapolli et al. 32. Model accuracy was assessed by calculating an $L_{2}$ norm of the difference between the LBM and the analytic results. The results in Table 2 summarise the $L_{2}$ norm observed at the coarsest grid spacing and the average order of convergence as the grid spacing was refined. A positive value for convergence indicates that the $L_{2}$ norm decreased with a reduction in grid spacing. As can be observed in these results, and in Figure 5 for Case 1, the passive scalar model struggled to capture the temperature-dependence within the Couette flow. The total energy model, however, captured the complex temperature-dependent interactions accurately for a range of parameter values. The cause of this is due to the passive scalar model only being able 


\begin{tabular}{|c|c|c|c|c|c|c|c|c|}
\hline & \multicolumn{4}{|c|}{ Passive Scalar } & \multicolumn{4}{c|}{ Total Energy } \\
\hline & \multicolumn{2}{|c|}{ Fluid } & \multicolumn{2}{c|}{ Temperature } & \multicolumn{2}{c|}{ Fluid } & \multicolumn{2}{c|}{ Temperature } \\
\hline Case & $L_{2}$ & Order & $L_{2}$ & Order & $L_{2}$ & Order & $L_{2}$ & Order \\
\hline 1 & $3.1 \mathrm{e}-3$ & -0.2 & $5.5 \mathrm{e}-2$ & 0.0 & $2.4 \mathrm{e}-3$ & 2.0 & $3.9 \mathrm{e}-5$ & 1.8 \\
2 & $3.4 \mathrm{e}-3$ & -0.2 & $4.7 \mathrm{e}-2$ & 0.0 & $3.9 \mathrm{e}-3$ & 2.0 & $9.0 \mathrm{e}-5$ & 1.6 \\
3 & $9.6 \mathrm{e}-3$ & -0.1 & $1.4 \mathrm{e}-1$ & 0.0 & $1.5 \mathrm{e}-3$ & 2.1 & $2.0 \mathrm{e}-4$ & 2.2 \\
4 & $2.1 \mathrm{e}-3$ & 0.2 & $2.3 \mathrm{e}-2$ & 0.0 & $2.7 \mathrm{e}-3$ & 2.0 & $1.4 \mathrm{e}-5$ & 1.6 \\
5 & $6.1 \mathrm{e}-4$ & 0.0 & $9.1 \mathrm{e}-2$ & 0.0 & $1.8 \mathrm{e}-5$ & 2.0 & $1.4 \mathrm{e}-5$ & 2.0 \\
\hline
\end{tabular}

Table 2: $L_{2}$ norm results for the coarsest grid spacing and the approximate order of convergence for the tested LBM models with temperature-dependent viscosity when compared to the analytic results. A positive value for convergence indicates that the $L_{2}$ decreased with a reduction in grid spacing.

to generate a linear temperature field between points of different temperature. The consequence is that features that are generated by more complex heating modes, shear heating for example, are not resolved in this model. This is re- 


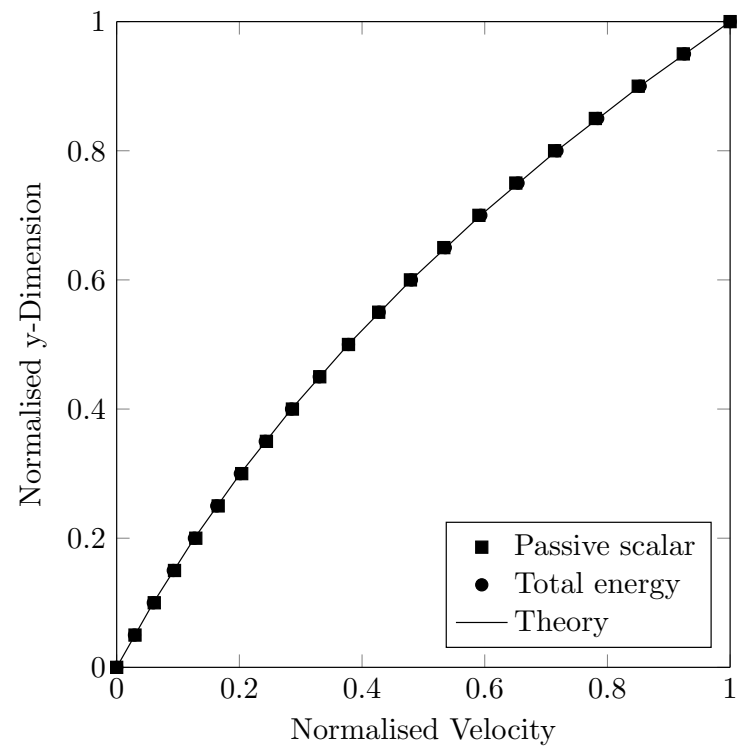

(a) Normalised velocity profile

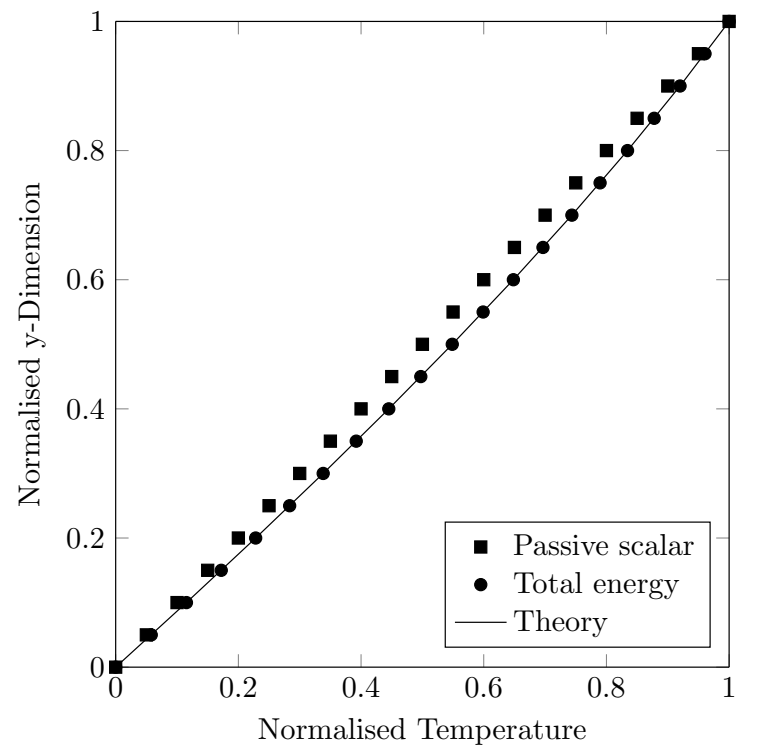

(b) Normalised temperature profile

Figure 5: An example comparison of the passive scalar (squares) and total energy (circles) LBM models with the analytic results of Myers et al. 31 for the normalised velocity and temperature profiles generated within a temperature-dependent Couette flow and applied temperature gradient. 


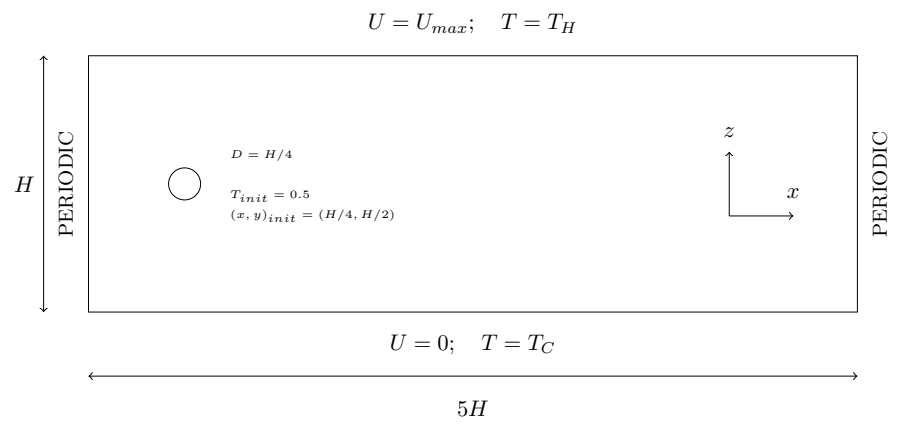

Figure 6: To investigate particle motion in a Couette flow with temperature-dependent viscosity, the verification test layout had a single particle placed in the centre of the channel. It was allowed to move freely with the flow. These tests were conducted with $H=40$. Fluid conditions were initialised as per Figure 4

\subsubsection{Single particle transport}

The first test consisted of a single particle located within the centre of channel experiencing Couette flow. It was constructed in a non-dimensional frame of reference. As per the verification test, the lower (stationary) wall was maintained at $T_{C}=0$ and the upper (moving) wall was held at $T_{H}=1$. This particle was prescribed a diameter of $D=H / 4$, where $H$ is the channel height, and an initial temperature of 0.5. It started at rest and was allowed to move freely with the flow. Fluid conditions were initialised as per Figure 4. These tests were conducted with a channel height of $H=40$ and thus a particle diameter of 10 lattice spacings. This layout is presented in Figure 6. This simulation was run for two cases, the first with the LBM viscosity varying exponentially with local fluid temperature as per the verification tests and the second with it being held constant at the lower wall value. This compared the impact of variable and constant viscosity on particle motion. This pair of simulations was also repeated with the temperature of the upper and lower boundaries swapped. This was used to determine whether the temperature effects on viscosity or the hydrodynamics of the Couette flow field was the primary contributor to particle motion. 

locity and vertical displacement of the particle was tracked. The normalised results of these are presented in Figure 7. Here it can be observed that after the initial state of rest had been overcome, the particle in the variable viscosity case migrated at a constant rate towards the upper boundary. By the end of the simulation the particle had moved a distance of $0.17 D$ (approximately $0.0425 H)$. In comparison, when the viscosity is held constant the particle effectively remains in the same position in the centre of the channel. On initial inspection this can be attributed to the thinner (lower viscosity) fluid closer to the hot wall reducing the stress experienced by the particle on its upper surface previous case. The magnitude of particle velocity is also significantly greater with the temperature boundary conditions swapped.

Both of these observations can be associated with the change in temperature and velocity profiles that occur due to the temperature boundary conditions compared to the lower surface. This imbalance causes the observed migration of the particle. It can also be noted that due to the relatively minor vertical motion of the particle in the constant viscosity case that, for the parameters in use in this study, hydrodynamic effects on particle motion are limited.

A similar argument can be made for the reduced magnitude of particle velocity in the variable viscosity case. In the centre of the channel, the viscosity of the fluid surrounding the particle in the variable viscosity case is less than that experienced with the constant viscosity case (which is taken at the cold lower wall value). This thinner fluid is unable to accelerate the particle as effectively and as such it takes longer to achieve a steady-state velocity matching that of the local flow.

In the simulations with the temperature conditions swapped (Figure 7), it can be seen from the vertical motion that the particle has again migrated towards the region of lower viscosity. It can also be noted here that in Figure 7 the constant viscosity results are identical and can be used as a reference. In the swapped layout, particle migration has occurred at a slower rate than in the changing in the original Couette flow. This is illustrated in Figure 8. In partic- 


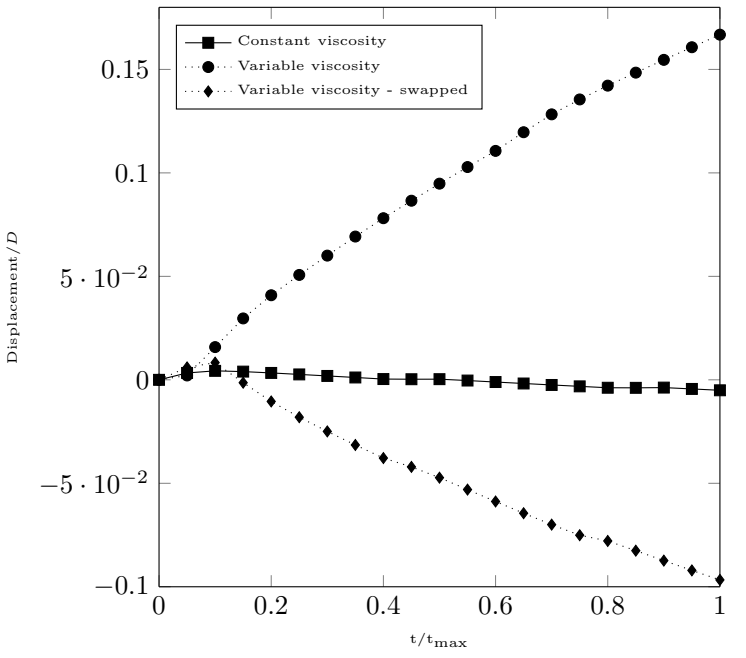

(a) Normalised particle vertical displacement

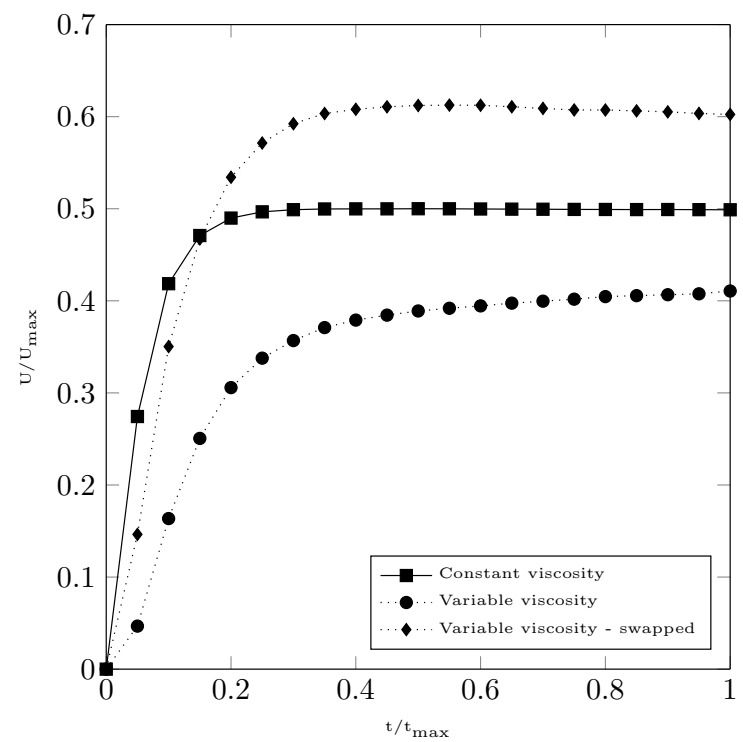

(b) Normalised particle velocity magnitude

Figure 7: Comparison of the vertical displacement (normalised by particle diameter) and velocity magnitude (normalised by wall velocity) of a single particle within a Couette flow influenced by a temperature gradient. The constant viscosity results (squares) represent a fluid with no temperature dependence. A temperature-dependent fluid is represented for the original temperature gradient as depicted in Figure 6 (circles) and when the temperature boundaries are swapped (diamonds). 
ular, the fluid velocity in the second case is significantly faster over the entire height of the channel. This observation is due to the higher viscosity fluid being located at the moving plane of the upper wall. In the first case, the hot and thin fluid was less capable of transferring shear stress as a result it did not effectively accelerate the fluid below it. In the second case, the motion of the thick fluid by the wall is more readily able to accelerate the adjacent fluid layers and this continues over the height of the channel. The result is that the particle in the second case is driven by a higher local fluid velocity than when the viscosity is held constant, allowing it to achieve a greater overall velocity. The greater momentum of the particle along the length of the channel may also hinder vertical motion contributing to the reduced rate of migration observed in the second case.

\subsection{Model application - 3D channel flows}

A 3D version of this model was implemented using the open-source frameworks of TCLB (LBM, 33]) and ESyS-Particle (DEM, 34]). This framework was used to study the transport of particle suspensions of various solid volume fractions within a fluid with temperature-dependent viscosity.

Channel flows with a number of geometric variations were selected as the test cases for the developed model as they can be representative of multiple engineering and scientific problems. These can be simplifications of pipe networks in industrial processing, hydraulic fractures within oil and gas extraction or blood vessels in biology. The three cases considered here are a straight channel, a straight channel with a side channel where fluid and particles can leak from, and finally a stepped channel. In all three cases, the channel is periodic in the vertical direction with gravity applied parallel to the periodic axis. Particles are continuously injected along the full height of the channel at the inlet of the domain with a horizontal velocity of $U_{\text {inject }}=0.1 \mathrm{~m} / \mathrm{s}$ and the same initial solid volume fraction (SVF). Particles are added to the domain when the leading $w$ is vacant of particles. When dimensionless temperature is applied to wall bound- 


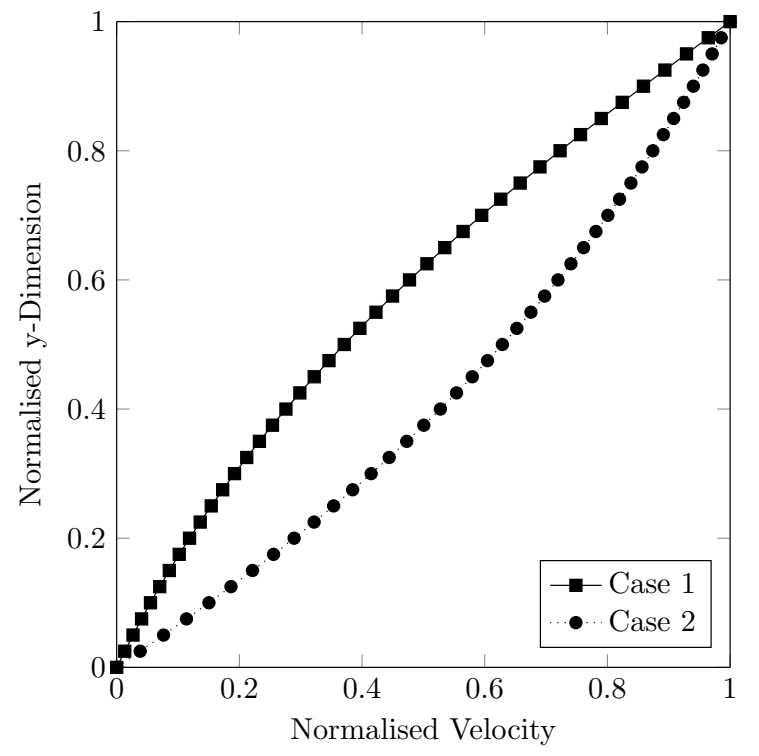

(a) Normalised velocity profile

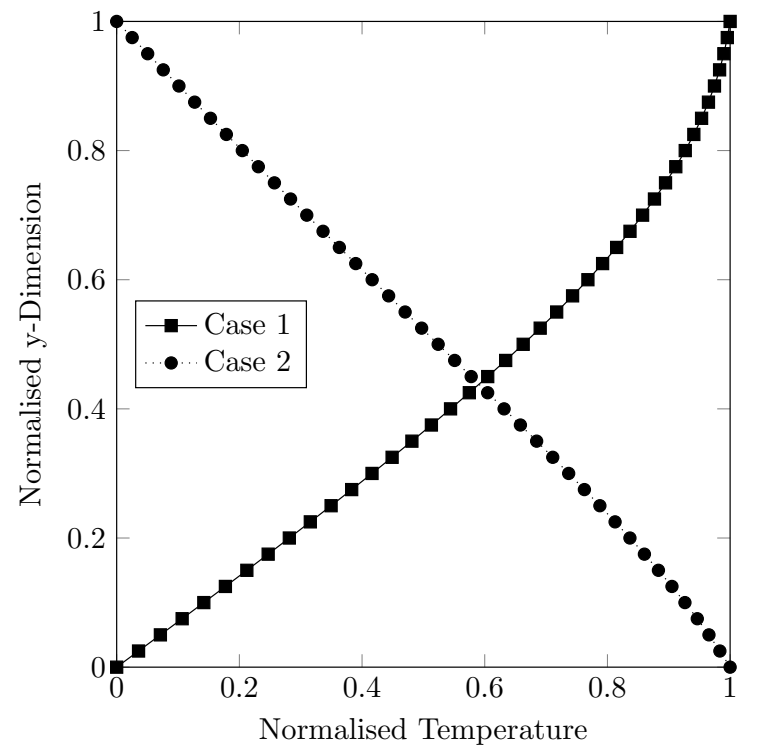

(b) Normalised temperature profile

Figure 8: Normalised velocity and temperature profiles observed within a Couette flow when the temperature boundary conditions are swapped (Case 2) from their original configuration (Case 1). 
outlet along the main channel direction. From a physical perspective, these can be interpreted based on $T_{H}^{\text {physical }}=350 \mathrm{~K}$ and $T_{C}^{\text {physical }}=300 \mathrm{~K}$. Bulk fluid is generally initialised at $U_{\text {inject }}$ in the x-direction and at a temperature of $T^{\text {lattice }}$ $=0.5$. $U_{\text {inject }} / 2$ was used in the leaking channel as due to there being two exits

365 for fluid from the domain. $U_{\text {inject }}$ was maintained at the boundary in this case. Fluid on the inlet face were always injected at $U_{\text {inject }}$. In all cases tested here, the LBM spacing has been set at $\Delta x=3.125 \times 10^{-5} \mathrm{~m}$ with a time step of $\Delta t=$ $40 \mu \mathrm{s}$, this corresponds to a kinematic viscosity of $\nu=4.07 \times 10^{-5} \mathrm{~m}^{2} / \mathrm{s}$ when a numerical viscosity of $1 / 6$ is used. Unless noted otherwise, the particles used in these studies have a radius of $r=6.4 \Delta x=0.0002 m$ (diameter $D=0.0004 m$ ). The particle injection zone is taken as $w=64 \Delta x=0.002 \mathrm{~m}$. Initial particles began with a velocity of $U_{\text {inject }}$ in the x-direction and a temperature of $T^{\text {lattice }}$ $=0.5$. Particles in the 'Step' section of the stepped channel began moving in the z-direction.

\subsubsection{Straight channel}

The model domain to investigate the effects of temperature-dependent viscosity on the settling behaviour of particles within a straight channel is presented in Figure 9. The fluid entered with fixed velocity and exited with a constant pressure boundary condition implemented using the well known Zou-He method [35. This model was run for a total of 250,000 steps.

To compare the settling behaviour of the two cases, the particle velocities averaged along the length of the channel were computed. These results after 125,000 and 250,000 steps are presented in Figure 10 Firstly, it can be noted that the velocity along the length of the channel (x-direction) is essentially unchanged by the introduction of temperature-dependent viscosity. The magnitude of this is tending around the injection viscosity as expected from mass conservation. An equilibrium state for this appears to have been reached after 250,000 steps. The variation between the data points is dependent on how many particles were located around that position at the time of interest.

The most interesting measure, though, is the vertical (y-direction) velocity. In 


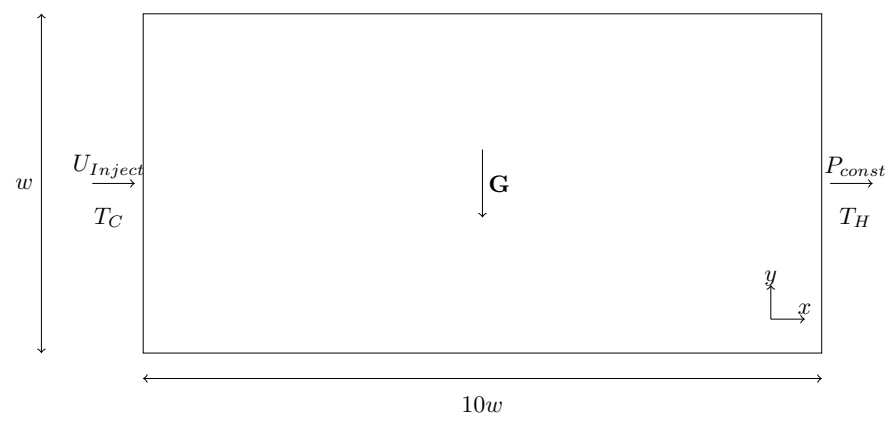

Figure 9: Layout of settling within a straight channel geometry. The entire model has a thickness of $w$. The model is initially filled with particles of diameter $D=0.2 w$, these are continuously injected with the inlet velocity into the leading $w$ of the domain. The upper and lower boundaries in the $y$ direction are periodic.

the absence of changing viscosity, this slowly increases along the length of the channel due to the action of gravity. When a temperature-dependent viscosity is introduced, the magnitude of the settling velocity increases by a factor of approximately three. Similar trends were observed throughout the simulation, rather than just the example times given here. The distribution of particles and their velocities after 250,000 steps is presented in Figure 11. In particular, the increase in settling velocity can be noted in this figure. Similar trends of behaviour can be observed when a higher initial SVF (33\% compared to 8\%) of particles was inserted into the channel geometry (Figure 12p. In this case, the magnitude of increase in vertical velocity is about a factor of two by the end of the channel. However, the increased number of collisions between particles around the entrance to the channel would be suggested as the reason why particles have not reached an equilibrium velocity at the entrance after 250,000 steps. The distribution of particles and their velocities after 250,000 steps is illustrated more graphically in Figure 13. Again, the increase in settling velocity can be noted here. 

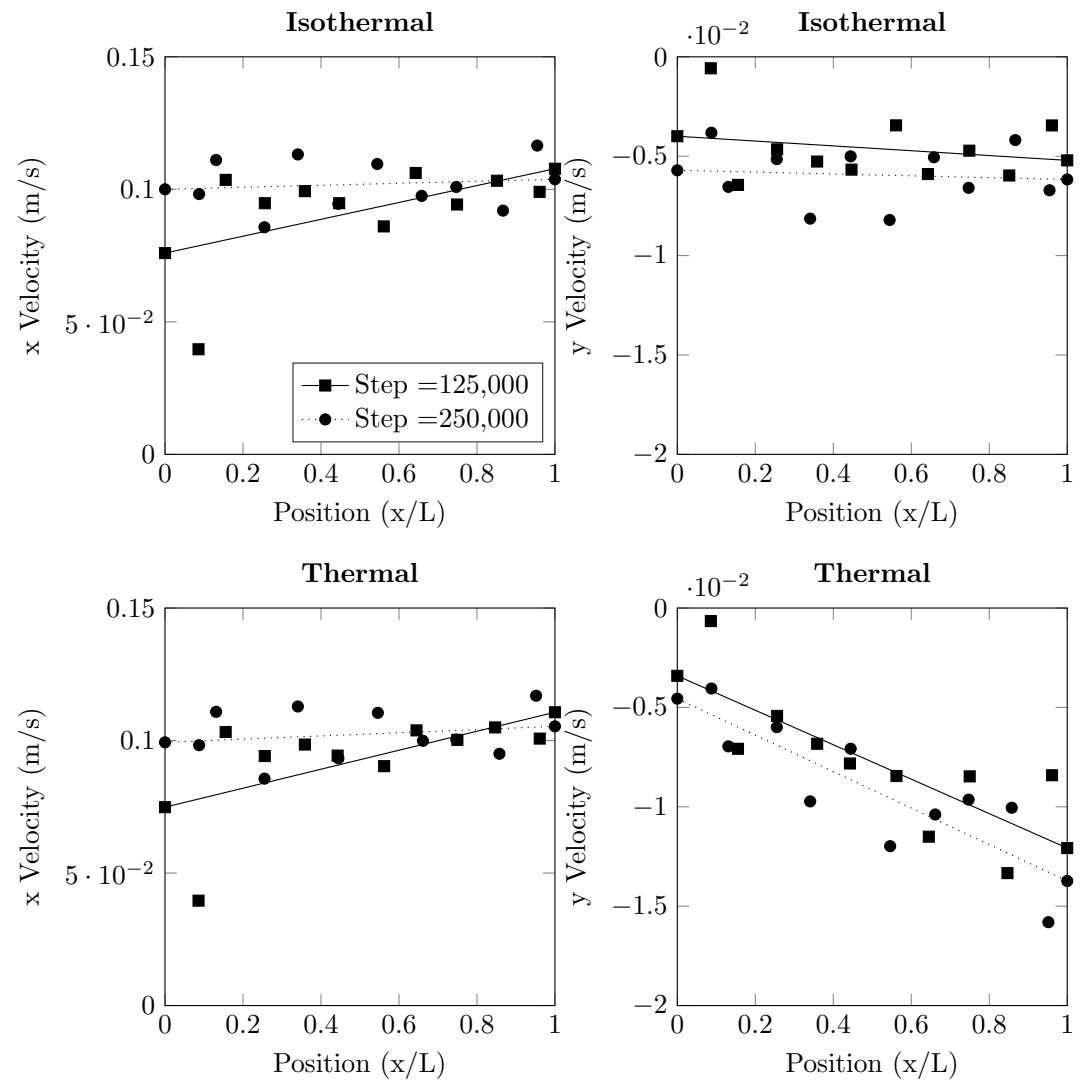

Figure 10: Velocity of the particles in the $\mathrm{x}$ - and $\mathrm{y}$-directions averaged along the length of the straight channel ( $8 \%$ initial SVF) recorded at 125,000 and 250,000 steps. The isothermal plots have constant viscosity whilst the thermal results have temperature-dependent viscosity. The line represents a least-squares fit to the data points. 

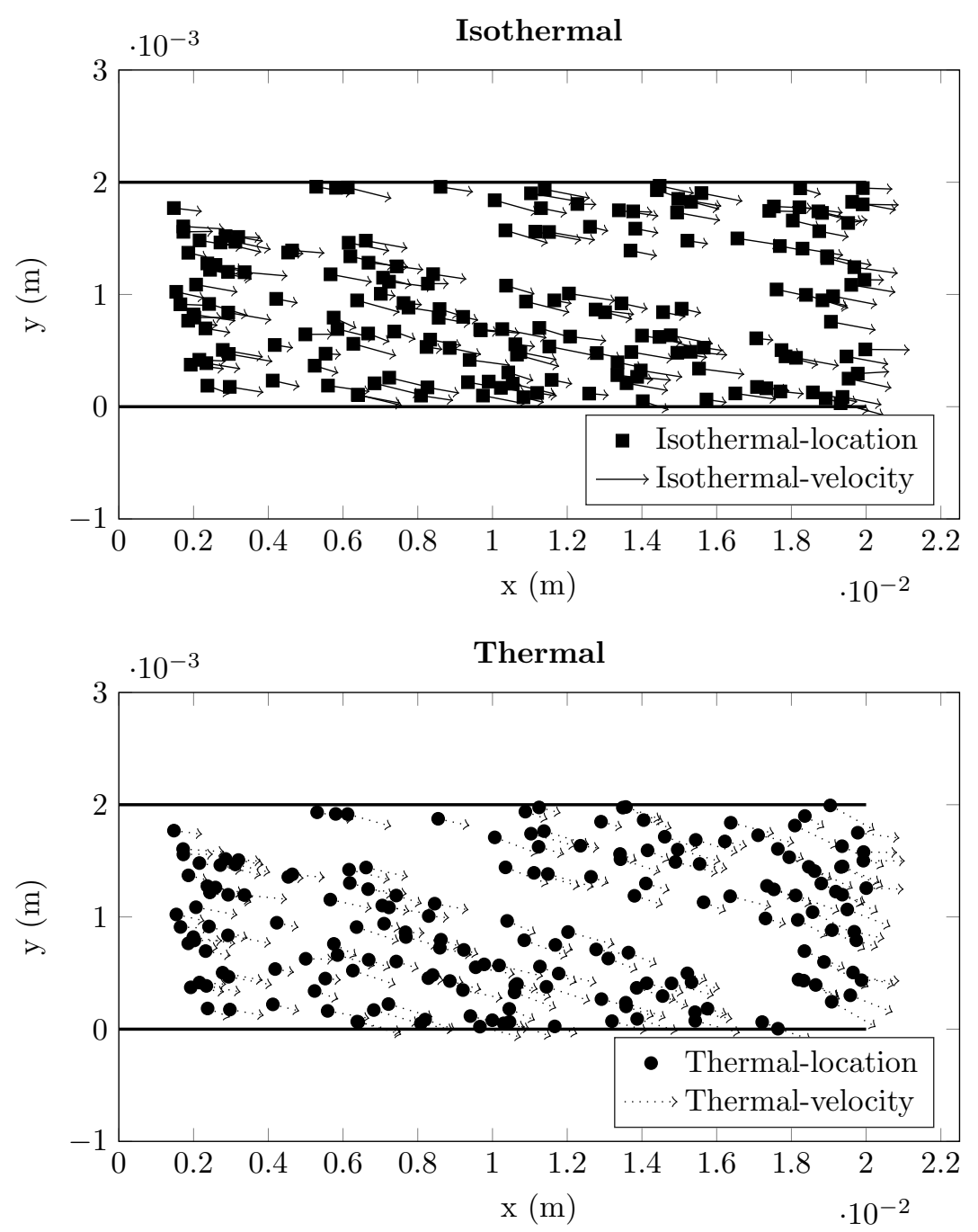

Figure 11: Comparison of the particle position and velocity vectors of the particles in a straight channel under both temperature conditions (8\% initial SVF) recorded at 250,000 steps. The isothermal plots have constant viscosity whilst the thermal results have temperaturedependent viscosity. The increase in settling velocity can be noted in the Thermal case. 

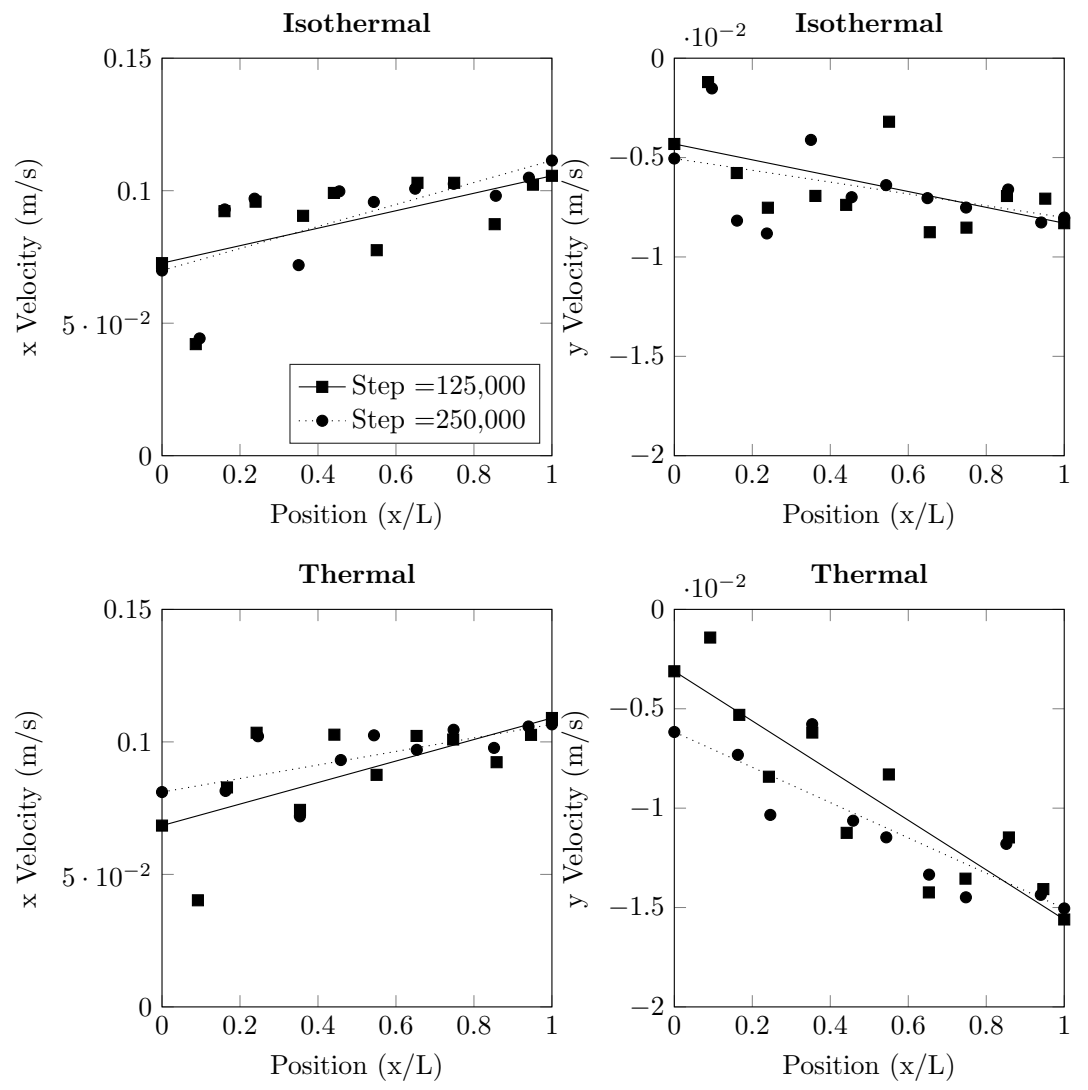

Figure 12: Velocity of the particles in the $\mathrm{x}$ - and $\mathrm{y}$-directions averaged along the length of the straight channel filled with a $33 \%$ initial SVF of particles. Data here is recorded after 125,000 and 250,000 steps. The isothermal plots have constant viscosity whilst the thermal results have temperature-dependent viscosity. The line represents a least-squares fit to the data points. 

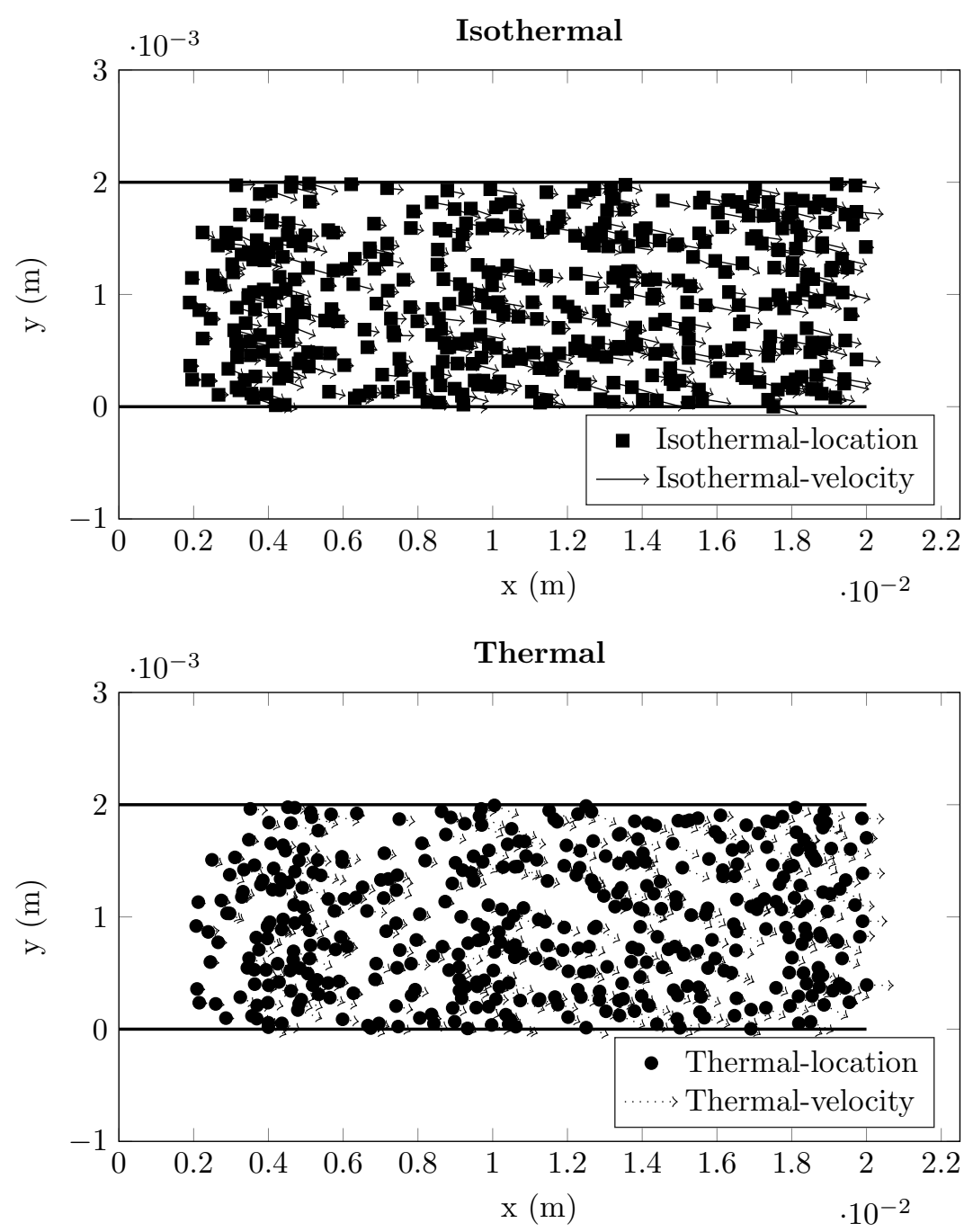

Figure 13: Comparison of the particle position and velocity vectors of the particles under both temperature conditions (33\% initial SVF) recorded at 250,000 steps. The isothermal plots have constant viscosity whilst the thermal results have temperature-dependent viscosity. The increase in settling velocity can be noted in the Thermal case. 


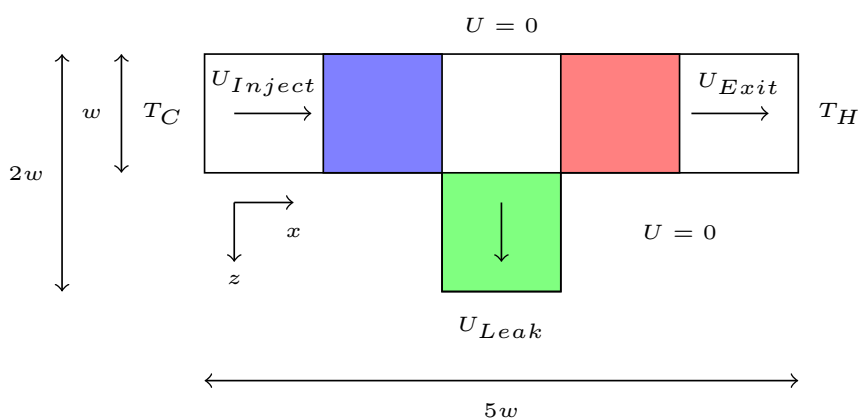

Figure 14: Layout for flow through a leaking channel geometry. Each flow channel has a thickness of $w$. The model is initially filled with particles of diameter $D=0.2 w$, these are continuously injected with the inlet velocity into the leading $w$ of the domain. The upper and lower boundaries in the y-direction (out-of-page) are periodic. The width and flow rates of the leak and main channel are adapted to investigate their impact on flow. The blue section represents the 'Entering' section, green the 'Leaking' and red the 'Continuing' sections used for counting particles.

\subsubsection{Leaking channel}

The study of a leaking channel, as described in Figure 14 involved narrowing the leaking pathway such that its width was reduced to $2.5 \mathrm{D}$ and $1.25 \mathrm{D}$ with pressure boundaries at the exits. All tests were performed for 100,000 time steps. The initial SVF of these cases was approximately $26 \%$.

As part of the analysis of both these cases, three key regions of the channel domain were identified. The 'Entering' section is the region of length $w$ immediately prior to the beginning of the leak channel, the 'Leaking' section is the entire volume of the side channel and the 'Continuing' section is the region of length $w$ immediately after the leak channel. The cumulative total of particles moving through the domain was recorded to infer the passage of particles in a simulation.

When a changing channel width was implemented to regulate flow through the leaking channel, variation was observed between the isothermal and thermal results. These can be observed in Figures 15, 16, 17 and 18. In Figure 15 it can be readily observed that the restriction of flow caused by the narrowed leak 
channel has made the main channel the strongly preferred path for the flow of particles. In the case of the width being $1.25 \mathrm{D}$, the 'Continuing' region has slightly more particles than the 'Entering' region due to the simulation being initialised fully packed with particles. A number of these will begin between these to regions and contribute to the 'Continuing' count and not the 'Entering' one. The greater rate of leaking in the case of the wider channel overcame this in that case. Halving the channel width led to a proportionally greater drop in particles being extracted on the leaking pathway. In the wider channel the simulation concluded with over 200 particles travelling through this region, this reduced to less than 50 particles in the narrower channel. In Figure 16, the effect of the thermal model can be seen on the difference in leaking rate between the two channels. In both cases, the presence of temperature-dependent viscosity corresponds to a significant reduction in the number of particles travelling along the leaking channel. In the wider channel, the observed reduction is approximately $10 \%$ by the end of the simulation, whereas a $22 \%$ reduction is noted in the narrow channel. A possible explanation for this is that the reduced viscosity of the fluid, and corresponding reduced drag on the particle, makes it more difficult for a particle to change direction and travel along a narrow leaking pathway. The velocity vector plots in Figures 17 and 18 highlight that only the particles very close to the leak channel are fed into the channel. It also appears that those particles in the leaking channel during the thermal case may have a reduced velocity compared to their isothermal counterparts. This would further reduce the flow rate of particles moving through the channel in this case. The case of the narrow leaking channel was repeated with the radius of particles halved, as a result the particles are of the same proportion compared to the wider leaking channel. This change caused the initial SVF to increase slightly to approximately $31 \%$. The lattice spacing again remained the same as in the original case. It can be observed that the smaller particles have a reduced tendency to be extracted away from the main channel through examination of Figures 19 and 20 As for the larger particles, the presence of temperature-dependent viscosity reduces the number of particles travelling along the 'Leaking' path. The 
reduction is about $10 \%$, similar to that seen in the wider channel for the larger particles. In Figure 21 it can be noted that the Poiseuille profile formed by the fluid dominates the distribution of particles through the channel. Particles become distributed along the walls in both temperature cases and this serves to delay injection of further particles.

None of the leaking channel cases presented here indicated the likelihood of forming a blockage due to a bridge forming across the mouth of the channel. This may be due to the flow rate through the main channel being too large or quantity of particles injected being too low for this to occur in a stable fashion. A second possibility is that the larger $3 \mathrm{D}$ height of the domains tested here meant that any such blockages were not able to fully seal off the leaking pathway. In 2D, or very low thickness $3 \mathrm{D}$ simulations, such behaviour may occur more readily.

\subsubsection{Stepped channel}

The final case study presented here examines the flow of particles with distributed size flowing through the domain outlined in Figure 22, Cases of low $(26 \%)$ and high (46\%) initial SVF of particles with a range of diameters $(D \in[0.2 w, 0.4 w]=[0.0004 m, 0.0008 m])$ were examined to investigate the changes in flow produced by this change. A pressure exit condition was used at the outlet of the domain. As for the leaking channel test, three regions can be identified: 'Entering' section is the region prior to the beginning of direction change, 'Step' section is the entire width of the domain where the direction change occurs and the 'Continuing' section is the region beyond the step change. Again, these were run for 100,000 steps. The significant direction change experienced by particles in this domain was observed to lead to increased overlap between particles and walls that was not observed in the previous test cases. To alleviate this overlap, the contact stiffness between both particles and walls was increased by a factor of 10, which still allowed stable DEM contact.

During these simulations, it was observed that the number of particles in the 'Entering' region of the stepped channel was less smooth in its evolution 

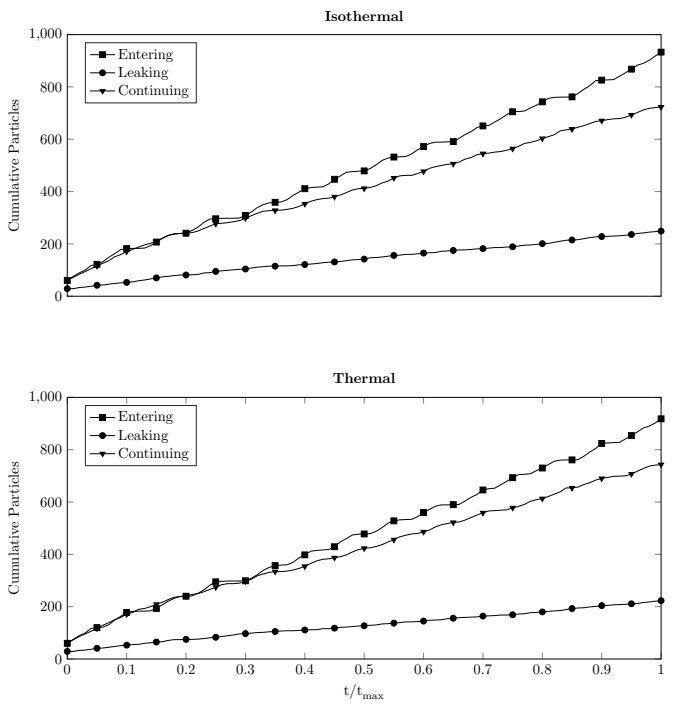

(a) Leak channel width $=2.5 \mathrm{D}$
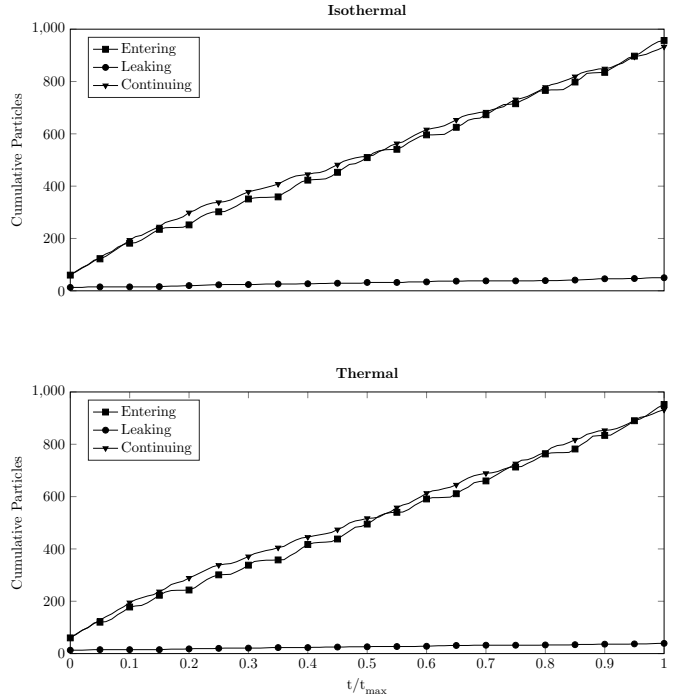

(b) Leak channel width $=1.25 \mathrm{D}$

Figure 15: Cumulative totals of particles moving through the three characteristic sections of the channel over the duration of simulations with differing leak widths. 

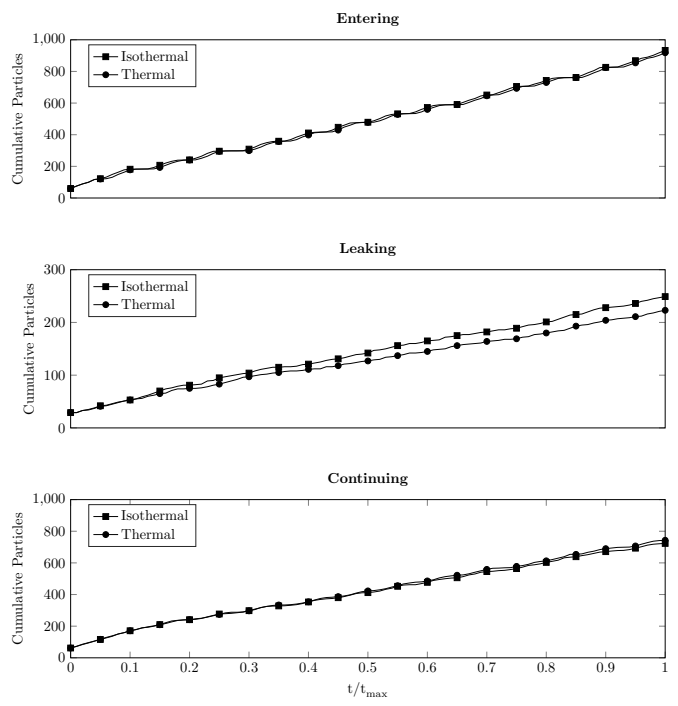

(a) Leak channel width $=2.5 \mathrm{D}$
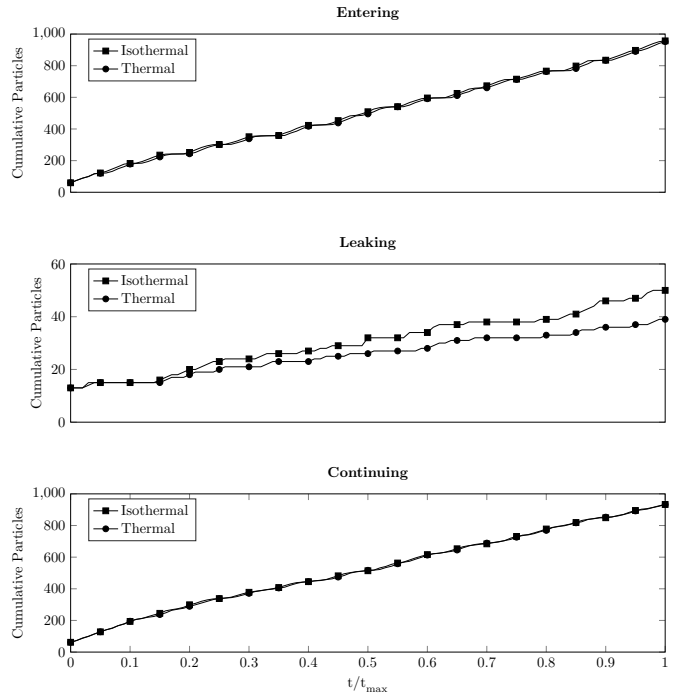

(b) Leak channel width $=1.25 \mathrm{D}$

Figure 16: Comparison of cumulative totals of particles in each of the three flow cases over the duration of simulations with differing leak channel widths. For each temperature case the differing behaviour in each of the characteristic regions can be observed. 


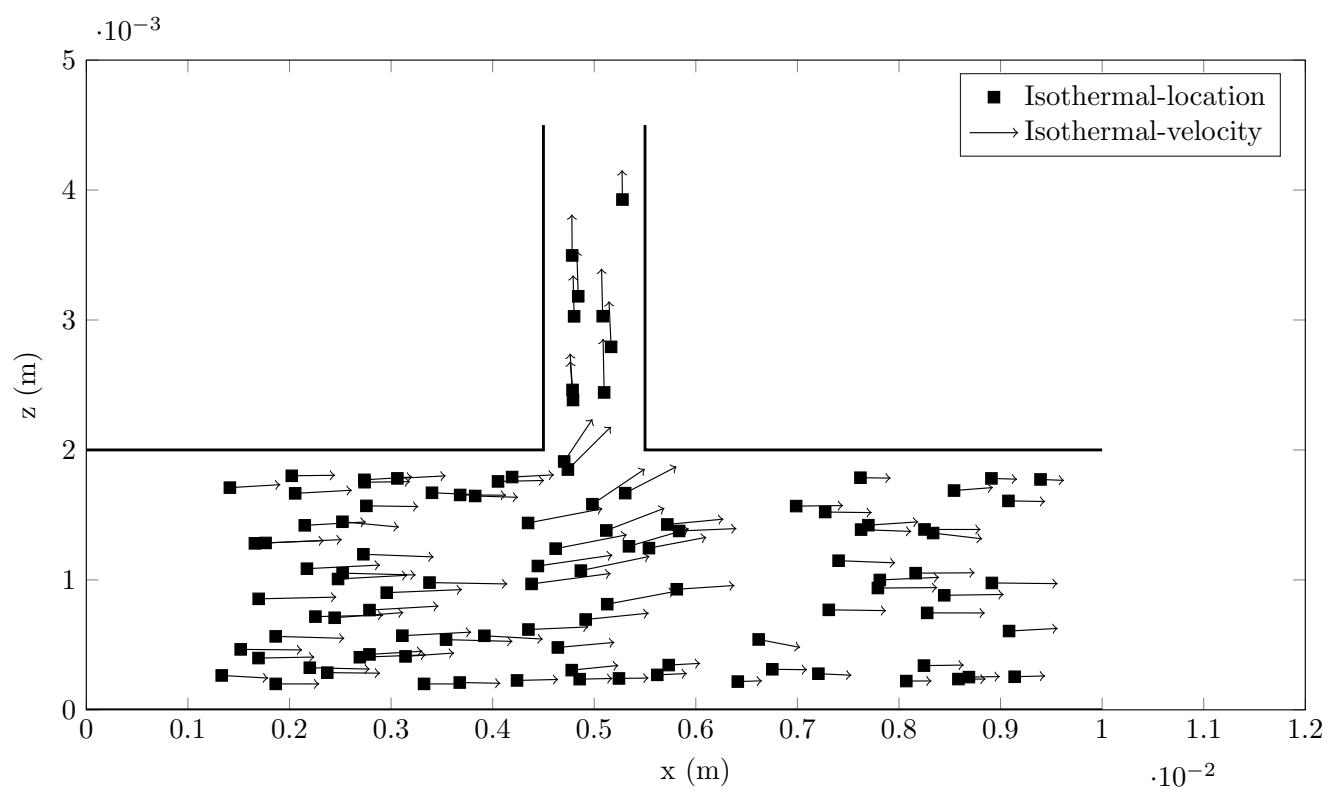

(a) Isothermal

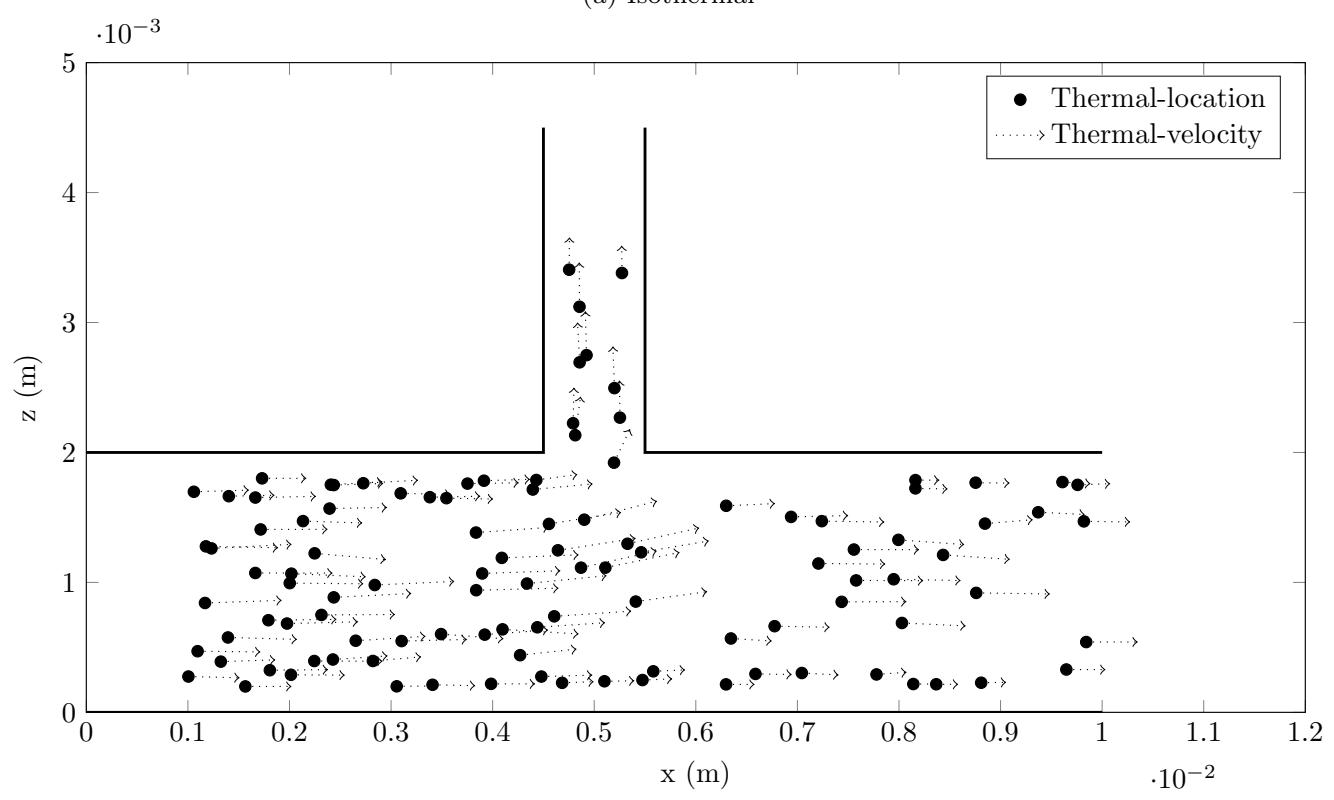

(b) Thermal

Figure 17: Comparison of the particle position and velocity vectors after the 100,000 time steps for each temperature case and leak channel width of $2.5 \mathrm{D}$. 


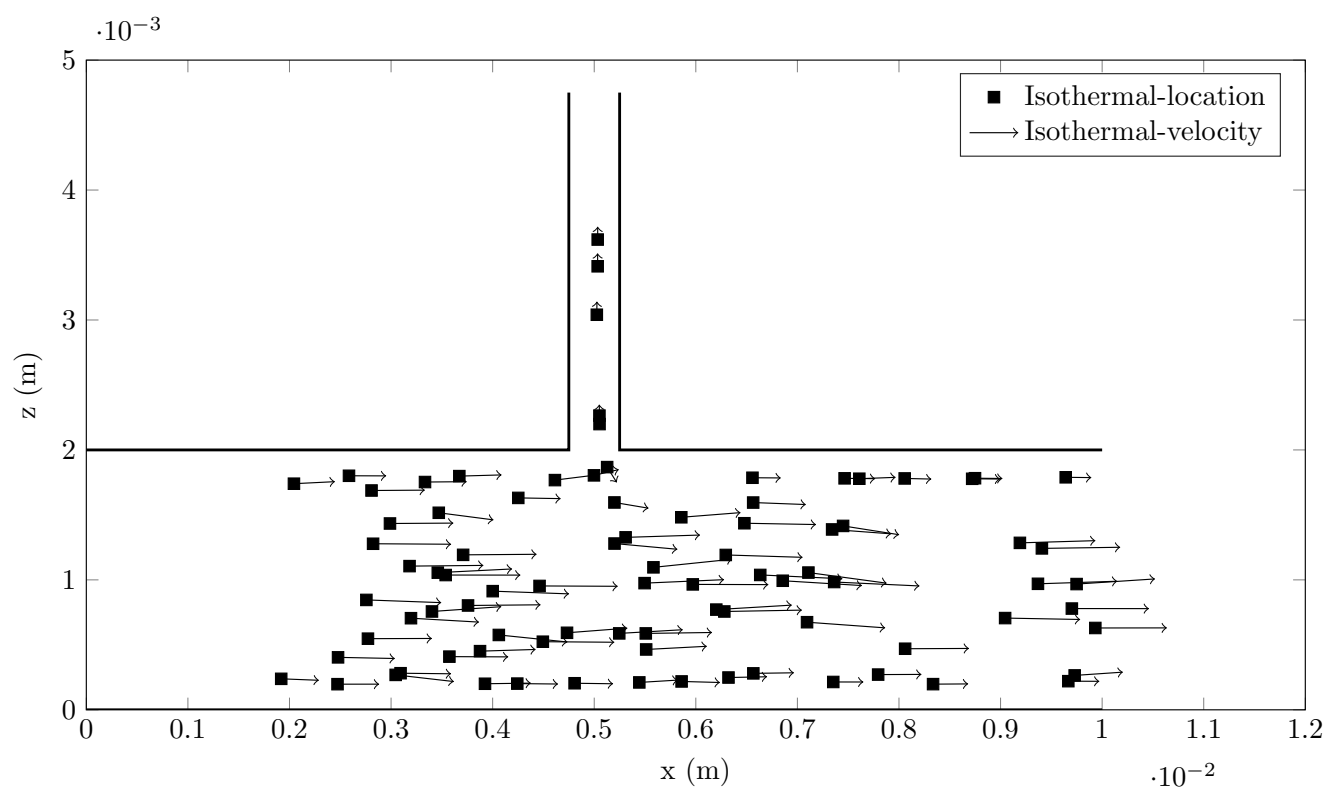

(a) Isothermal

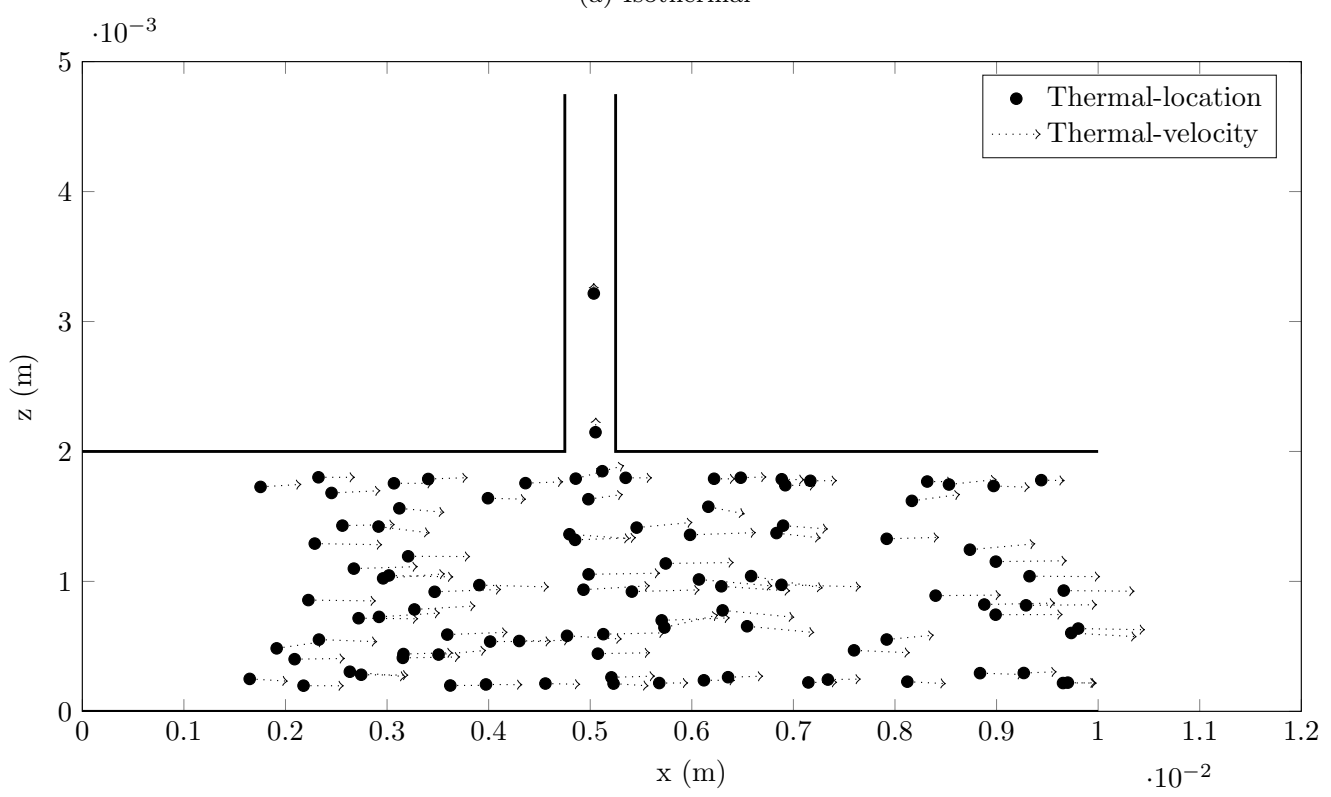

(b) Thermal

Figure 18: Comparison of the particle position and velocity vectors after the 100,000 time steps for each temperature case and leak channel width of $1.25 \mathrm{D}$. 

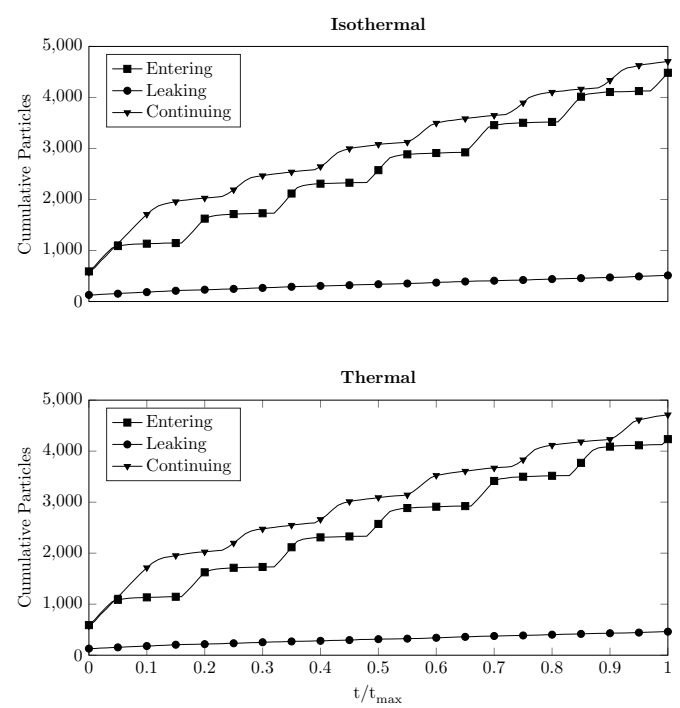

Figure 19: Cumulative totals of particles moving through the three characteristic sections of the channel over the duration of simulations for the narrow leaking channel with smaller particles.
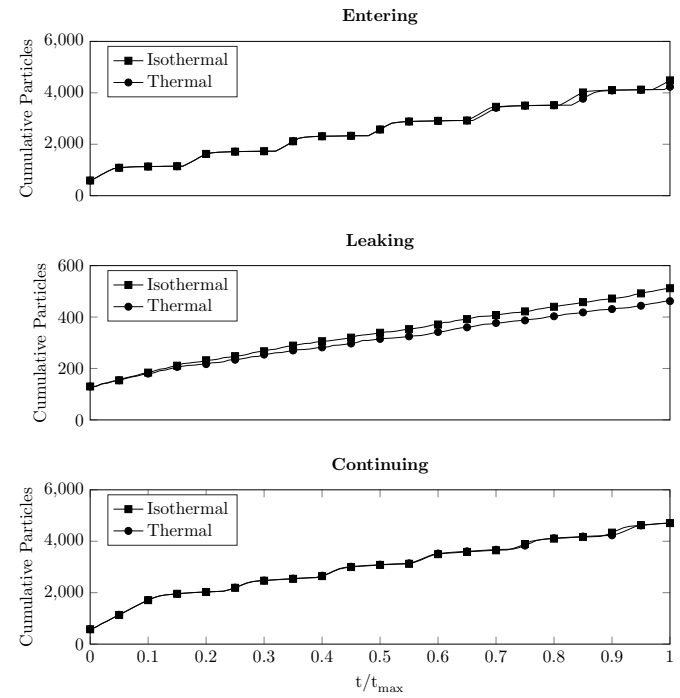

Figure 20: Comparison of cumulative totals of particles in each of the three flow cases over the duration of simulations for the narrow leaking channel with smaller particles. For each temperature case the differing behaviour in each of the characteristic regions can be observed. 


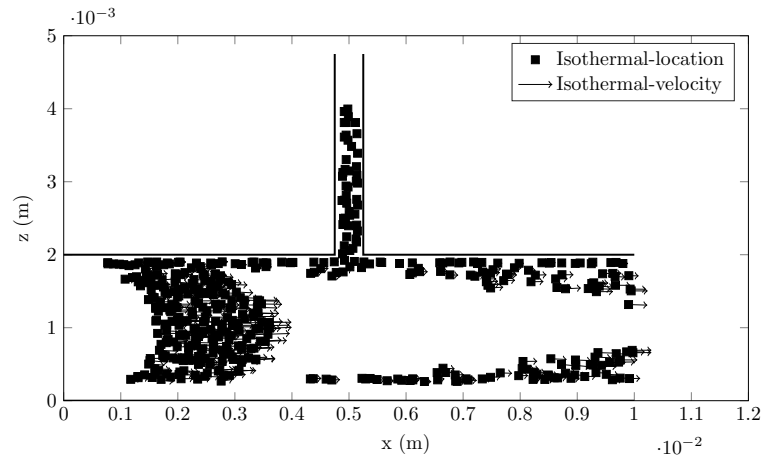

Figure 21: Comparison of the particle position and velocity vectors after the 100,000 time steps for each temperature case for the narrow leaking channel with smaller particles. Only isothermal results are shown here. The dominating Poiseuille profile causes particles to remain trapped on the edges of the domain, this serves to delay further injection of particles.

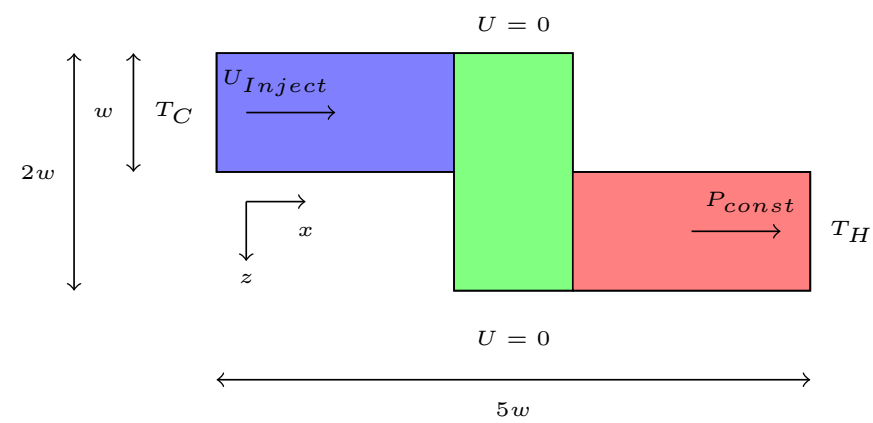

Figure 22: Layout for flow through a stepped channel geometry. The flow channel has a thickness of $w$. The model is initially filled with particles with diameters distributed in the range $D \in[0.2 w, 0.4 w]$, these are continuously injected with the inlet velocity into the leading $w$ of the domain. The upper and lower boundaries in the $y$ direction are periodic. The blue, green and red regions represent the 'Entering', 'Step' and 'Continuing' regions respectively for counting particles. 
than compared to the other two regions. This is a consequence of the injection of particles not happening consistently through the simulation. Instead, the algorithm waits until the injection area is clear of previous particles before a new batch is inserted into the flow. Due to the nature of Poiseuille flow, it was observed that smaller particles would occasionally be caught in an area of low-speed flow near the walls, delaying further injection of particles. The more consistent gradient of the plots in the high SVF case suggests that the greater bulk of particles in this case is better able to overcome any potential blockages or entrainment of particles caused by the change in direction within the domain. This behaviour is made clear in the velocity vector plots in Figure 23. In the low SVF case, the transition of particle direction through the direction change is much smoother than seen in the high SVF case. This allows for pockets of the channel $($ e.g. near $(x, y)=(0.006 m, 0.000 m)$ and $(0.004 m, 0.004 m))$ to be empty of particles as they follow the natural fluid flow path through the domain. In the high SVF case, particles in these regions can be observed to collide with and follow the wall as they move through the 'Step' region. In both cases, a much greater velocity magnitude can be observed in particles moving through the constriction provided by the neck of the step change.

\section{Summary}

This paper has focussed on the modelling of particle suspension flows within a fluid with temperature-dependent viscosity using a coupled LBM-DEM framework. It was firstly demonstrated that, in order to correctly capture the fundamental behaviour of such fluids, a total energy formulation of the thermal LBM is required. The commonly used passive scalar approach is unable to resolve non-linear temperature profiles generated due to temperature-dependent viscosity. The developed model is extended to 3D and applied to numerous examples of channel flow. The case studies presented illustrate how a temperaturedependent viscosity fluid can generate quantitative differences in the transport of particles through a domain. In many practical applications, the efficient 


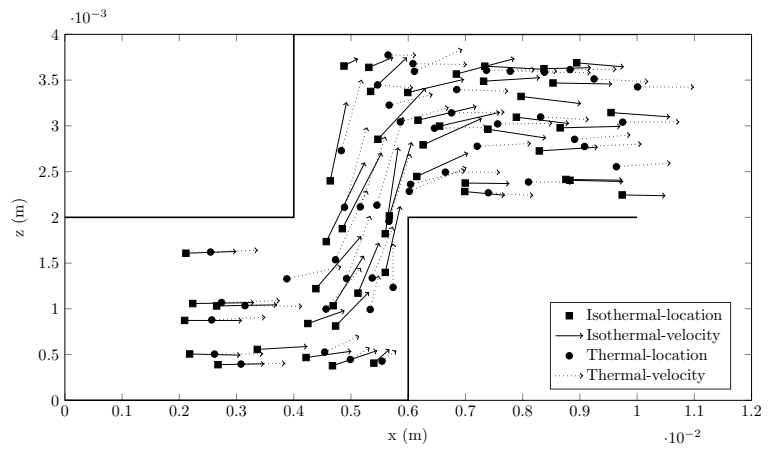

(a) Low SVF (26\%)

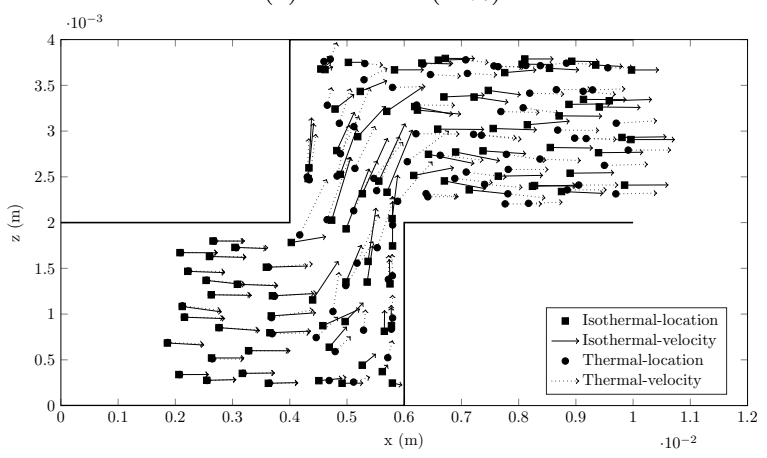

(b) High SVF (46\%)

Figure 23: Comparison of the particle position and velocity vectors after the 100,000 time steps for each temperature case and each initial SVF in the stepped domain. 
transport of particles by the fluid is key to system performance. If the phenomena highlighted in these studies (e.g. increased settling velocity and reduced leak-off behaviour) are not properly considered by operators than inefficient or indeed ineffective operation may result.

The model presented in this paper still contains a number of areas where it can be improved as part of continued work. In particular, to allow for the study of larger sets of particles the coupling of the TCLB and ESyS-Particle codes can be made more computationally efficient. The introduction of lubrication force interactions between particles would also provide a more physically realistic representation of particle transport both within confined channels and in dense flows. Similarly, the expansion of this work to non-spherical particles would allow the model to be applied in a wider variety of scientific and industrial 525 scenarios. This study has been entirely numerical, experimental study of some of the cases presented in Section 3.3 would provide additional strength to the results presented in this work.

All fluids possess temperature-dependent viscosity. This study has demonstrated that neglecting the effect of this can lead to quantitative changes in the behaviour of the fluid and particles transported within in. We have also demonstrated that modelling this behaviour accurately requires specific considerations to be made. Overlooking this behaviour can generate inefficiencies in practical applications in a number of engineering and scientific fields such as oil and gas production, chemical processing and cooling systems.

\section{Acknowledgements}

Support of this work through the Australian Government Research Training Program Scholarship, the UQ School of Mechanical and Mining Engineering, the UQ Graduate School International Travel Award and the Australian Petroleum Production and Exploration Association (K.A. Richards Scholarship) are all gratefully acknowledged by the authors. The iRMB group at Technische Universität Braunschweig are also sincerely thanked for hosting JM in the process 
of completing this research. This work was supported by resources provided by The Pawsey Supercomputing Centre with funding from the Australian Government and the Government of Western Australia. We thank the anonymous reviewers for their comments and suggestions for improving this manuscript.

\section{References}

„ [1] I. V. Karlin, D. Sichau, S. S. Chikatamarla, Consistent two-population lattice Boltzmann model for thermal flows, Physical Review E 88 (2013) 063310. doi:10.1103/PhysRevE.88.063310 URL http://link .aps .org/doi/10.1103/PhysRevE.88.063310

n [2] X. He, S. Chen, G. D. Doolen, A novel thermal model for the lattice Boltzmann method in incompressible limit, Journal of

घ Computational Physics 146 (1) (1998) 282 - 300. doi:http: //dx.doi.org/10.1006/jcph.1998.6057.

555 URL http://wWw.sciencedirect.com/science/article/pii/ S0021999198960570

q [3] P. Lallemand, L.-S. Luo, Hybrid finite-difference thermal lattice Boltzmann equation, International Journal of Modern Physics B 17 (1-2) (2003)

1 41-47. arXiv:http://www.worldscientific.com/doi/pdf/10.1142/ S0217979203017060, doi:10.1142/S0217979203017060. URL http://www.worldscientific.com/doi/abs/10.1142/ S0217979203017060

口 [4] A. Vikhansky, Construction of lattice-Boltzmann schemes for nonNewtonian and two-phase flows, The Canadian Journal of Chemical Engineering 90 (5) (2012) 1081-1091. doi:10.1002/cjce.21664

URL http://dx.doi.org/10.1002/cjce.21664

q [5] C. Leonardi, D. Owen, Y. Feng, Numerical rheometry of bulk materials using a power law fluid and the lattice Boltzmann method Journal of Non-Newtonian Fluid Mechanics 166 (12) (2011) 628 - 638. 
doi:https://doi.org/10.1016/j.jnnfm.2011.02.011.

口

URL http://www.sciencedirect.com/science/article/pii/ S0377025711000772

a 6] S. Gabbanelli, G. Drazer, J. Koplik, Lattice Boltzmann method for non-

q newtonian (power-law) fluids, Physical Review E 72 (2005) 046312. doi:

$575 \quad 10.1103 /$ PhysRevE.72.046312.

URL https://link.aps.org/doi/10.1103/PhysRevE.72.046312

[7] Z. Guo, T. Zhao, Lattice Boltzmann simulation of natural convection with temperature-dependent viscosity in a porous cavity, Progress in Computational Fluid Dynamics $5(1 / 2)(2005)$.

${ }_{580}^{\text {口 }}$ [8] Z. Guo, C. Zheng, B. Shi, T. S. Zhao, Thermal lattice Boltzmann equation for low Mach number flows: Decoupling model, Physical Review E 75 (2007) 036704. doi:10.1103/PhysRevE.75.036704.

URL http://link.aps.org/doi/10.1103/PhysRevE.75.036704

q [9] X.-R. Zhang, Y. Cao, A lattice Boltzmann model for natural convection with a large temperature difference, Progress in Computational Fluid Dynamics, an International Journal 11 (5) (2011) 269278. arXiv:https://www.inderscienceonline.com/doi/pdf/10.1504/ PCFD.2011.042179, doi:10.1504/PCFD.2011.042179.

प URL https://www.inderscienceonline.com/doi/abs/10.1504/PCFD. 2011.042179

[10] Y. Cao, Variable property-based lattice Boltzmann flux solver for thermal flows in the low Mach number limit, International Journal of Heat and Mass Transfer 103 (2016) $254 \quad$ - 264. doi:https://doi.org/10.1016/j.ijheatmasstransfer.2016.07.052. URL http://www.sciencedirect.com/science/article/pii/ S0017931016312078

[11] P. L. Bhatnagar, E. P. Gross, M. Krook, A model for collision processes in 口. gases. I. Small amplitude processes in charged and neutral one-component 
URL http://link.aps.org/doi/10.1103/PhysRev.94.511

[12] G. McNamara, A. Garcia, B. Alder, Stabilization of thermal lattice Boltzmann models, Journal of Statistical Physics 81 (1-2) (1995) 395-408. doi:10.1007/BF02179986,

[13] C. Levermore, Moment closure hierarchies for kinetic theories, Journal of Statistical Physics 83 (5-6) (1996) 1021-1065. doi:10.1007/BF02179552. URL http://dx.doi.org/10.1007/BF02179552

[14] D. d'Humieres, Generalized lattice Boltzmann equations, in: B. Shizgal, D. Weaver (Eds.), Rarefied gas dynamics: theory and simulations, Vol. 159 of Progress in Astronautics and Aeronautics, AIAA, 1992, pp. 450-458.

[15] D. d'Humieres, I. Ginzburg, M. Krafczyk, P. Lallemand, L. Luo, Multiplerelaxation-time lattice Boltzmann models in three dimensions, Philosophical Transactions of the Royal Society of London A: Mathematical, Physical and Engineering Sciences 360 (1792) (2002) 437-451. doi:10.1098/rsta. 2001.0955 .

[16] Y. H. Qian, D. D'Humieres, P. Lallemand, Lattice BGK models for NavierStokes equation, Europhysics Letters 17 (6) (1992) 479.

URL http://stacks .iop.org/0295-5075/17/i=6/a=001

[17] T. Kruger, H. Kusumaatmaja, A. Kuzmin, O. Shardt, G. Silva, E. Viggen, The Lattice Boltzmann Method: Principles and Practice, Springer, 2017. doi:10.1007/978-3-319-44649-3.

[18] J. Williams, R. O'Connor, Discrete element simulation and the contact problem, Archives of Computational Methods in Engineering 6 (4) (1999) 279-304. doi:10.1007/BF02818917.

URL http://dx.doi.org/10.1007/BF02818917 
[19] E. Onate, J. Rojek, Combination of diescrete element and finite element methods for dynamic analysis of geomechanics problems, Computer Methods in Applied Mechanics and Engineering 193 (2004) 3087-3128.

[20] K. Walayat, Z. Zhang, K. Usman, J. Chang, M. Liu, Fully resolved simulations of thermal convective suspensions of elliptic particles using a multigrid fictitious boundary method, International Journal of Heat and Mass Transfer 139 (2019) 802-821.

[21] B. K. Cook, D. R. Noble, J. R. Williams, A direct simulation method for particle-fluid systems, Engineering Computations 21 (2/3/4) (2004) 151168.

[22] A. J. C. Ladd, Numerical simulations of particulate suspensions via a discretized Boltzmann equation. Part 1. Theoretical foundation, Journal of Fluid Mechanics 271 (1994) 285-309. doi:10.1017/S0022112094001771. URL http://journals . cambridge.org/article_S0022112094001771

[23] A. J. C. Ladd, Numerical simulations of particulate suspensions via a discretized Boltzmann equation. Part 2. Numerical results, Journal of Fluid Mechanics 271 (1994) 311-339. doi:10.1017/S0022112094001783. URL http://journals .cambridge.org/article_S0022112094001783

645

[24] K. Suzuki, T. Inamuro, Effect of internal mass in the simulation of a moving body by the immersed boundary method, Computers \& Fluids 49 (1) (2011) 173 - 187. doi:https://doi.org/10.1016/j.compfluid.2011.05.011. URL http://www.sciencedirect.com/science/article/pii/ S0045793011001708

[25] D. R. Noble, J. R. Torczynski, A lattice-Boltzmann method for partially saturated computational cells, International Journal of Modern Physics C 9 (8) (1998) 1189-1201.

[26] A. Xu, L. Shi, T. Zhao, Thermal effects on the sedimentation behavior of 
elliptical particles, International Journal of Heat and Mass Transfer 126 (2018) 753-764.

[27] A. Xu, W. Shyy, T. Zhao, Lattice Boltzmann modeling of transport phenomena in fuel cells and flow batteries, Acta Mech. Sin. 33 (3) (2017) 555-574. doi:10.1007/s10409-017-0667-6.

[28] B. D. Jones, J. R. Williams, Fast computation of accurate spherecube intersection volume, Engineering Computations 34 (4) (2017) 12041216. arXiv:https://doi.org/10.1108/EC-02-2016-0052, doi:10. 1108/EC-02-2016-0052

URL https://doi .org/10.1108/EC-02-2016-0052

[29] D. Wang, C. R. Leonardi, S. M. Aminossadati, Improved coupling of 665 time integration and hydrodynamic interaction in particle suspensions using the lattice Boltzmann and discrete element methods, Computers \& Mathematics with Applications 75 (7) (2018) 2593 - 2606. doi:https://doi.org/10.1016/j.camwa.2018.01.002.

1) URL http://wWw.sciencedirect.com/science/article/pii/ S0898122118300063

[30] O. E. Strack, B. K. Cook, Three-dimensional immersed boundary conditions for moving solids in the lattice-Boltzmann method, International Journal for Numerical Methods in Fluids 55 (2) (2007) 103-125.

URL http://dx.doi.org/10.1002/fld.1437

${ }_{675}^{[31]}$ T. Myers, J. Charpin, M. Tshehla, The flow of a variable viscosity fluid between parallel plates with shear heating, Applied

1. Mathematical Modelling 30 (9) (2006) 799 - 815. doi:https: //doi.org/10.1016/j.apm.2005.05.013.

1 URL http://www.sciencedirect.com/science/article/pii/ S0307904X05001125

[32] N. Frapolli, S. S. Chikatamarla, I. V. Karlin, Multispeed entropic lattice Boltzmann model for thermal flows, Physical Review E 90 (2014) 043306. 
doi:10.1103/PhysRevE.90.043306

URL http://link.aps.org/doi/10.1103/PhysRevE.90.043306

685

[33] TCLB Reference Manual (2019).

URL https://docs.tclb.io/

[34] ESyS-Particle Community Wiki (2019).

URL http://www.esys-particle.org/wiki/

[35] Q. Zou, X. He, On pressure and velocity boundary conditions for the lattice

690 Boltzmann BGK model, Physics of Fluids 9 (6) (1997) 1591-1598. doi: http://dx.doi.org/10.1063/1.869307.

प

URL http://scitation.aip.org/content/aip/journal/pof2/9/6/10.

$1063 / 1.869307$ 